\title{
Multifractal Spectra of Random Self-Affine Multifractal Sierpinski Sponges in $\mathbb{R}^{d}$
}

\author{
J.M. FRASER \& L. OlSEN
}

\begin{abstract}
In this paper we study the Hausdorff multifractal spectrum of random self-affine multifractal Sierpinski sponges in $\mathbb{R}^{d}$.
\end{abstract}

\section{Statements of Results}

In this paper we study the Hausdorff multifractal spectrum of random self-affine multifractal Sierpinski sponges in $\mathbb{R}^{d}$. The Hausdorff multifractal spectrum of a measure is defined in Section 1.1. In Section 1.2 we recall the definition of classical (non-random) self-affine multifractal Sierpinski sponges in $\mathbb{R}^{d}$ introduced by Bedford [B] and McMullen [M2] in the mid-1980s, and we introduce various notation that will be used later. Finally, in Section 1.3 we define random self-affine multifractal Sierpinski sponges in $\mathbb{R}^{d}$ and state our main results, providing almost sure formulas for the Hausdorff multifractal spectrum of certain classes of random self-affine multifractal Sierpinski sponges.

1.1. The Hausdorff multifractal spectrum. For a probability measure $\mu$ on $\mathbb{R}^{d}$ (or on a general metric space) with support equal to $K$, we define the Hausdorff multifractal spectrum function, $f_{\mathrm{H}, \mu}$, of $\mu$ by

$$
f_{\mathrm{H}, \mu}(\alpha)=\operatorname{dim}_{\mathrm{H}}\left\{x \in K \mid \lim _{r>0} \frac{\log \mu(B(x, r))}{\log r}=\alpha\right\},
$$

for $\alpha \geq 0$, where $\operatorname{dim}_{H}$ denotes the Hausdorff dimension. It is equally natural to consider the packing multifractal spectrum function, $f_{\mathrm{P}, \mu}$, of $\mu$ defined by

$$
f_{\mathrm{P}, \mu}(\alpha)=\operatorname{dim}_{\mathrm{P}}\left\{x \in K \mid \lim _{r \backslash 0} \frac{\log \mu(B(x, r))}{\log r}=\alpha\right\},
$$

for $\alpha \geq 0$, where dimp denotes the packing dimension. 
During the 1990s there was an enormous interest in computing the multifractal spectra of measures in the mathematical literature, and within the last 10-15 years the multifractal spectra of various classes of measures in Euclidean space $\mathbb{R}^{d}$ exhibiting some degree of self-similarity have been computed rigorously; cf. [F2] and the references therein.

Regrettably, the fractal and multifractal structure of self-affine sets and measures is significantly less well understood. This is clearly due to the very substantial difficulties arising from the fact that affine maps contract with different amounts in different directions, and not a question of lack of interest. In fact, self-affine sets and measures appear naturally in the study of dynamical systems. Namely, attractors and natural occupation measures on attractors of nonconformal dynamical systems are locally self-affine. Because of this, there has within the past 10-15 years been an enormous interest in the mathematical literature in studying the fractal properties of self-affine sets; cf., for example, the papers [AN, B, F1, F3, FW, GL1, K, KP, M2, O1, P, R2, T] and the excellent survey paper by Peres and Solomyak [PS].

However, in addition to investigating the fractal properties of self-affine sets, much more recently there has been a significant and substantially increased interest in understanding the fractal and multifractal properties of self-affine measures. Indeed, in the late 90s King $[\mathrm{K}]$ and Olsen [O1] computed the multifractal spectrum of self-affine Sierpinski sponges [K, O1], and within the past 2-3 years a number of papers investigating this and related problems have appeared; see, for example, [BM1, BM2, F3, GL2, GL3, GL4, GL5, GL6, JS, JR, N, O2, T]. Despite this, the multifractal spectra of self-affine measures are still not well understood, and the multifractal spectra have only been investigated for a very limited number of non-random self-affine measures; indeed, all of the above mentioned papers (with the exception of [GL1]) investigate non-random self-affine sets and measures. In this paper we consider the more complicated problem of determining the Hausdorff multifractal spectrum of certain random versions of the self-affine Sierpinski sponges investigated in [BM1, BM2, GL2, GL3, GL4, GL5, GL6, JR, N, O2, T]. There are several reasons why an understanding of the multifractal structure of more sophisticated random self-affine constructions, such as the ones considered in this paper, are useful. Firstly, it may contribute towards a fuller understanding of the multifractal structure of general self-affine measures. Secondly, more sophisticated constructions may provide better models for the local self-affineness exhibited by many natural measures on attractors of non-conformal dynamical systems eventually leading to a better understanding of non-conformal dynamics.

We now give a brief description of the organization of the paper. In Section 1.2 we recall the definition of classical (non-random) self-affine multifractal Sierpinski sponges introduced by Bedford [B] and McMullen [M2] in the mid-1980s, and we introduce various auxiliary definitions and notation that will be used later. Section 1.3 is the main section of the paper. In this section we introduce random self-affine multifractal Sierpinski sponges and state our main results. Section 2 
contains examples illustrating our main results, and the proofs are given in Sections $3-7$.

\subsection{Self-affine multifractal Sierpinski sponges.}

1.2.1. Definition of self-affine multifractal Sierpinski sponges. We will now define self-affine Sierpinski sponges and introduce various auxiliary definitions and notation that will be used later. Let $d \in \mathbb{N}$ and

$$
\mathbf{n}=\left(n_{1}, \ldots, n_{d}\right)
$$

be a list of integers with

$$
1<n_{1} \leq n_{2} \leq \cdots \leq n_{d}
$$

and write

$$
\Sigma=\left\{0, \ldots, n_{1}-1\right\} \times \cdots \times\left\{0, \ldots, n_{d}-1\right\},
$$

i.e., $\Sigma$ denotes the family of vectors $\mathbf{i}=\left(i_{1}, \ldots, i_{d}\right)$ which have entries $i_{\ell} \in$ $\left\{0, \ldots, n_{\ell}-1\right\}$. For $\mathbf{i}=\left(i_{1}, \ldots, i_{d}\right) \in \Sigma$, define affine maps by $S_{\mathbf{i}}:[0,1]^{d} \rightarrow$ $[0,1]^{d}$ by

$$
S_{\mathbf{i}}\left(x_{1}, \ldots, x_{d}\right)=\left(\frac{1}{n_{1}} x_{1}+\frac{i_{1}}{n_{1}}, \ldots, \frac{1}{n_{d}} x_{d}+\frac{i_{d}}{n_{d}}\right) .
$$

Next, fix $D \subseteq \Sigma$, and let $\mathbf{p}=\left(p_{\mathbf{i}}\right)_{\mathbf{i} \in D}$ be a probability vector. Write

$$
\Sigma^{\mathbb{N}}=\left\{\mathbf{i}_{1} \mathbf{i}_{2} \cdots \mid \mathbf{i}_{j} \in \Sigma \text { for all } j\right\}
$$

for the family of all infinite strings $\mathbf{i}_{1} \mathbf{i}_{2} \ldots$ with entries $\mathbf{i}_{j} \in \Sigma$ and define $\pi$ : $\Sigma^{\mathbb{N}} \rightarrow[0,1]^{d}$ by

$$
\left\{\pi\left(\mathbf{i}_{1} \mathbf{i}_{2} \ldots\right)\right\}=\bigcap_{n} S_{\mathbf{i}_{1}} \cdots S_{\mathbf{i}_{n}}\left([0,1]^{d}\right) .
$$

The self-affine Sierpinski sponge $K_{\mathbf{n}, D}$ (associated with $\mathbf{n}$ and $D$ ) is now defined by

$$
K_{\mathbf{n}, D}=\left\{\pi\left(\mathbf{i}_{1} \mathbf{i}_{2} \ldots\right) \mid \mathbf{i}_{\ell} \in D \text { for all } \ell\right\},
$$

and the self-affine multifractal Sierpinski sponge $\mu_{\mathbf{n}, D, \mathbf{p}}$ (associated with $\mathbf{n}, D$ and p) is the unique probability measure such that

$$
\mu_{\mathbf{n}, D, \mathbf{p}}\left(S_{\mathbf{i}_{1}} \cdots S_{\mathbf{i}_{n}}\left([0,1]^{d}\right)\right)=p_{\mathbf{i}_{1}} \cdots p_{\mathbf{i}_{n}}
$$

for all positive integers $n$ and all finite strings $\mathbf{i}_{1} \ldots \mathbf{i}_{n}$ with $\mathbf{i}_{j} \in D$. 
1.2.2. The Hausdorff multifractal spectrum of self-affine multifractal Sierpinski sponges. In [O1] Olsen computed the Hausdorff multifractal spectrum of $\mu_{\mathbf{n}, D, \mathbf{p}}$. In order to state this result we need to introduce the following notation. This notation will also be used when stating the main results in the next section. For $\ell=0,1, \ldots, d$, define $\pi_{\ell}: \mathbb{R}^{d} \rightarrow \mathbb{R}^{\ell}$ by

$$
\pi_{\ell}\left(x_{1}, \ldots, x_{d}\right)=\left(x_{1}, \ldots, x_{\ell}\right)
$$

$\ell=1, \ldots, d$ and $\pi_{\ell}\left(x_{1}, \ldots, x_{d}\right)=0$ for $\ell=0$. Next, for $\ell=1, \ldots, d$ and $\left(i_{1}, \ldots, i_{\ell}\right) \in \pi_{\ell}(D)$, we define conditional probabilities $p_{D, \mathbf{p}, \ell}\left(i_{\ell} \mid i_{1}, \ldots, i_{\ell-1}\right)$ by

$$
p_{D, \mathbf{p}, \ell}\left(i_{\ell} \mid i_{1}, \ldots, i_{\ell-1}\right)=\frac{\sum_{\substack{\mathbf{j}=\left(j_{1}, \ldots, j_{d}\right) \in D \\ j_{1}=i_{1}, \ldots, j_{\ell}=i_{\ell}}} p_{\mathbf{j}}}{\sum_{\substack{\mathbf{j}=\left(j_{1}, \ldots, j_{d}\right) \in D \\ j_{1}=i_{1}, \ldots, j_{\ell-1}=i_{\ell-1}}} p_{\mathbf{j}}} .
$$

Finally, we define auxiliary functions $\beta_{\mathbf{n}, D, \mathbf{p}, i_{1} \ldots i_{d}}, \beta_{\mathbf{n}, D, \mathbf{p}, i_{1} \ldots i_{d-1}}, \ldots, \beta_{\mathbf{n}, D, \mathbf{p}, i_{1} i_{2}}$, $\beta_{\mathbf{n}, D, \mathbf{p}, i_{1}}, \beta_{\mathbf{n}, D, \mathbf{p}}: \mathbb{R} \rightarrow \mathbb{R}$ inductively as follows:

(0) For $q \in \mathbb{R}$ and $\left(i_{1}, \ldots, i_{d}\right) \in D$, define $\beta_{\mathbf{n}, D, \mathbf{p}, i_{1} \ldots i_{d}}(q)$ by

$$
\beta_{\mathbf{n}, D, \mathbf{p}, i_{1} \ldots i_{d}}(q)=0 .
$$

(1) For $q \in \mathbb{R}$ and $\left(i_{1}, \ldots, i_{d-1}\right) \in \pi_{d-1}(D)$, define $\beta_{\mathbf{n}, D, \mathbf{p}, i_{1} \ldots i_{d-1}}(q)$ by

$$
\sum_{\substack{i_{d} \\\left(i_{1}, \ldots, i_{d}\right) \in \pi_{d}(D)}} p_{D, \mathbf{p}, d}\left(i_{d} \mid i_{1}, \ldots, i_{d-1}\right)^{q} n_{d}^{\beta_{\mathbf{n}, D, p, i_{1} \ldots i_{d}}(q)-\beta_{\mathbf{n}, D, \mathbf{p}, i_{1} \ldots i_{d-1}}(q)}=1 .
$$

(2) For $q \in \mathbb{R}$ and $\left(i_{1}, \ldots, i_{d-2}\right) \in \pi_{d-2}(D)$, define $\beta_{\mathbf{n}, D, \mathbf{p}, i_{1} \ldots i_{d-2}}(q)$ by $\sum_{\substack{i_{d-1} \\\left(i_{1}, \ldots, i_{d-1}\right) \in \pi_{d-1}(D)}} p_{D, \mathbf{p}, d-1}\left(i_{d-1} \mid i_{1}, \ldots, i_{d-2}\right)^{q} n_{d-1}^{\beta_{\mathbf{n}, D, \mathbf{p}, i_{1} \ldots i_{d-1}}(q)-\beta_{\mathbf{n}, D, \mathbf{p}, i_{1} \ldots i_{d-2}}(q)}=1$.

(d) For $q \in \mathbb{R}$, define $\beta_{\mathbf{n}, D, \mathbf{p}}(q)$ by

$$
\sum_{\substack{i_{1} \\ i_{1} \in \pi_{1}(D)}} p_{D, \mathbf{p}, 1}\left(i_{1}\right)^{q} n_{1}^{\beta_{\mathbf{n}, D, \mathbf{p}, i_{1}}(q)-\beta_{\mathbf{n}, D, \mathbf{p}}(q)}=1 .
$$


Also, write

$$
\begin{aligned}
& \alpha_{\min , D, \mathbf{p}}=\min _{\left(i_{1}, \ldots, i_{d}\right) \in D}-\sum_{\ell} \frac{\log p_{D, \mathbf{p}, \ell}\left(i_{\ell} \mid i_{1}, \ldots, i_{\ell-1}\right)}{\log n_{\ell}}, \\
& \alpha_{\max , D, \mathbf{p}}=\max _{\left(i_{1}, \ldots, i_{d}\right) \in D}-\sum_{\ell} \frac{\log p_{D, \mathbf{p}, \ell}\left(i_{\ell} \mid i_{1}, \ldots, i_{\ell-1}\right)}{\log n_{\ell}} .
\end{aligned}
$$

In [O1] an explicit expression for the Hausdorff multifractal spectrum of $\mu_{\mathbf{n}, D, \mathbf{p}}$ is found assuming that $D$ satisfies the so-called Very Strong Separation Condition. We now define the Very Strong Separation Condition.

Definition (The Very Strong Separation Condition (VSSC)). A subset D of $\Sigma$ satisfies the Very Strong Separation Condition (VSSC) if it satisfies the following: if $\ell=1, \ldots, d$ and $\left(i_{1}, \ldots, i_{d}\right),\left(j_{1}, \ldots, j_{d}\right) \in D$ satisfy $i_{1}=j_{1}, \ldots, i_{\ell-1}=$ $j_{\ell-1}$ and $i_{\ell} \neq j_{\ell}$, then $\left|i_{\ell}-j_{\ell}\right|>1$.

We can now state the results from [O1], providing an explicit expression for the Hausdorff multifractal spectrum of $\mu_{\mathbf{n}, D, \mathbf{p}}$.

Theorem $\boldsymbol{A}$ ([O1]). Assume that D satisfies the VSSC.

(i) We have

$$
f_{\mathrm{H}, \mu_{\mathbf{n}, D, \mathbf{p}}}(\alpha)=\beta_{\mathbf{n}, D, \mathbf{p}}^{*}(\alpha)
$$

for all $\alpha \in\left(\alpha_{\min , D, \mathbf{p}}, \alpha_{\max , D, \mathbf{p}}\right)$ (here $\beta_{\mathbf{n}, D, \mathbf{p}}^{*}$ denotes the Legendre transform of $\beta_{\mathbf{n}, D, \mathbf{p}}$ defined by $\left.\beta_{\mathbf{n}, D, \mathbf{p}}^{*}(\alpha)=\inf _{q}\left(q \alpha+\beta_{\mathbf{n}, D, \mathbf{p}}(q)\right)\right)$.

(ii) We have

$$
\left\{x \in K_{\mathbf{n}, D} \mid \lim _{r \backslash 0} \frac{\log \mu_{\mathbf{n}, D, \mathbf{p}}(B(x, r))}{\log r}=\alpha\right\}=\emptyset
$$

for all $\alpha \notin\left[\alpha_{\min , D, \mathbf{p}}, \alpha_{\max , D, \mathbf{p}}\right]$.

Remark. Theorem A assumes the VSSC. This is clearly a very restrictive condition, and it would be very desirable if it could be omitted. Unfortunately, this problem seems particularly difficult. For example, it is only very recently that Jordan and Rams [JR] have been able to show that Theorem A remains true for $d=2$ even without assuming the VSSC. However, it is still not known whether the VSSC can be omitted for $d>2$.

\subsection{Random self-affine multifractal Sierpinski sponges.}

\subsubsection{Definition of random self-affine multifractal Sierpinski sponges.}

We will now define random versions of the self-affine sets and measures introduced in Section 1.2. Fix a positive integer $N$ and a probability vector $\left(p_{1}, \ldots, p_{N}\right)$. Write

$$
\Omega=\{1, \ldots, N\}^{\mathbb{N}},
$$


and then let $\mathbb{P}$ denote the natural product probability measure on $\Omega$ induced by $\left(p_{1}, \ldots, p_{N}\right)$, i.e.,

$$
\mathbb{P}=\prod_{\mathbb{N}} \sum_{i=1}^{N} p_{i} \delta_{i} .
$$

For each $i=1, \ldots, N$, let

$$
\mathbf{n}_{i}=\left(n_{i, 1}, \ldots, n_{i, d}\right)
$$

be a list of integers; observe that, contrary to the deterministic case (1.3), we are not assuming that the sequences $\left(n_{i, 1}, n_{i, 2}, \ldots, n_{i, d}\right)$ are increasing-instead we will only assume that the sequences $\left(n_{i, 1}, n_{i, 2}, \ldots, n_{i, d}\right)$ are increasing "on average"; see (1.14) below. Write

$$
v_{1}=\prod_{i} n_{i, 1}^{p_{i}}
$$

$$
v_{d}=\prod_{i} n_{i, d}^{p_{i}}
$$

i.e., $v_{\ell}$ is the weighted multiplicative average of the integers $n_{1, \ell}, \ldots, n_{N, \ell}$, and put

$$
\boldsymbol{v}=\left(v_{1}, \ldots, v_{d}\right) \text {. }
$$

We will now assume that

$$
v_{1}<v_{2}<\cdots<v_{d}
$$

loosely speaking, (1.14) says the sequences $\left(n_{i, 1}, \ldots, n_{i, d}\right)$ are increasing "on average”. Write

$$
\Sigma_{i}=\left\{0, \ldots, n_{i, 1}-1\right\} \times \cdots \times\left\{0, \ldots, n_{i, d}-1\right\} ;
$$

i.e., $\Sigma_{i}$ denotes the family of vectors $\mathbf{i}=\left(i_{1}, \ldots, i_{d}\right)$ which have entries $i_{\ell} \in$ $\left\{0, \ldots, n_{i, \ell}-1\right\}$. For $\mathbf{i}=\left(i_{1}, \ldots, i_{d}\right) \in \Sigma_{i}$, define affine maps by $S_{i, \mathbf{i}}:[0,1]^{d} \rightarrow$ $[0,1]^{d}$ by

$$
S_{i, \mathbf{i}}\left(x_{1}, \ldots, x_{d}\right)=\left(\frac{1}{n_{i, 1}} x_{1}+\frac{i_{1}}{n_{i, 1}}, \ldots, \frac{1}{n_{i, d}} x_{d}+\frac{i_{d}}{n_{i, d}}\right) .
$$

Next, fix

$$
D_{1} \subseteq \Sigma_{1}, \ldots, D_{N} \subseteq \Sigma_{N},
$$

and let

$$
\mathbf{p}_{1}=\left(p_{1, \mathbf{i}}\right)_{\mathbf{i} \in D_{1}}, \ldots, \mathbf{p}_{N}=\left(p_{N, \mathbf{i}}\right)_{\mathbf{i} \in D_{N}}
$$


be probability vectors.

For $\omega \in \Omega$, the random self-affine Sierpinski sponge $K_{\omega}$ and random selfaffine multifractal Sierpinski sponge $\mu_{\omega}$ are defined as follows. For each sequence $\omega=\left(\omega_{1}, \omega_{2}, \ldots\right) \in \Omega$, let

$$
\Sigma_{\omega}^{\mathbb{N}}=\left\{\mathbf{i}_{1} \mathbf{i}_{2} \ldots \mid i_{j} \in \Sigma_{\omega_{j}} \text { for all } j\right\}
$$

denote the family of all infinite strings $\mathbf{i}_{1} \mathbf{i}_{2} \ldots$ with entries $\mathbf{i}_{j} \in \Sigma_{\omega_{j}}$, and define $\pi_{\omega}: \sum_{\omega}^{\mathbb{N}} \rightarrow[0,1]^{d}$ by

$$
\left\{\boldsymbol{\pi}_{\omega}\left(\mathbf{i}_{1} \mathbf{i}_{2} \ldots\right)\right\}=\bigcap_{n} S_{\omega_{1}, \mathbf{i}_{1}} \cdots S_{\omega_{n}, \mathbf{i}_{n}}\left([0,1]^{d}\right) .
$$

Next, for each sequence $\omega=\left(\omega_{1}, \omega_{2}, \ldots\right) \in \Omega$, the random self-affine Sierpinski sponge $K_{\omega}$ (associated with $D_{1}, \ldots, D_{N}$ ) is defined by

$$
K_{\omega}=\left\{\pi_{\omega}\left(\mathbf{i}_{1} \mathbf{i}_{2} \ldots\right) \mid \mathbf{i}_{j} \in D_{\omega_{j}} \text { for all } j\right\},
$$

and the random self-affine multifractal Sierpinski sponge $\mu_{\omega}$ (associated with $D_{1}, \ldots, D_{N}$ and $\left.\mathbf{p}_{1}, \ldots, \mathbf{p}_{N}\right)$ is defined as the unique probability measure such that

$$
\mu_{\omega}\left(S_{\omega_{1}, \mathbf{i}_{1}} \cdots S_{\omega_{n}, \mathbf{i}_{n}}\left([0,1]^{d}\right)\right)=p_{\omega_{1}, \mathbf{i}_{1}} \cdots p_{\omega_{n}, \mathbf{i}_{n}}
$$

for all positive integers $n$ and all strings $\mathbf{i}_{1} \ldots \mathbf{i}_{n}$ with $\mathbf{i}_{j} \in D_{\omega_{j}}$. Observe that if $\mathbf{n}_{1}=\cdots=\mathbf{n}_{N}=\mathbf{n}, D_{1}=\cdots=D_{N}=D$ and $\mathbf{p}_{1}=\cdots=\mathbf{p}_{N}=\mathbf{p}$, then $K_{\omega}=K_{\mathbf{n}, D}$ and $\mu_{\omega}=\mu_{\mathbf{n}, D, \mathbf{p}}$ for all $\omega \in \Omega$. Also observe that if $N=1$ and we write $\mathbf{n}_{1}=\mathbf{n}, D_{1}=D$ and $\mathbf{p}_{1}=\mathbf{p}$, then $K_{\omega}=K_{\mathbf{n}, D}$ and $\mu_{\omega}=\mu_{\mathbf{n}, D, \mathbf{p}}$ for all $\omega \in \Omega$. Furthermore, it is not difficult to see that

$$
K_{\omega}=\lim _{n} \bigcup_{\mathbf{i}_{1} \in D_{\omega_{1}}, \ldots, \mathbf{i}_{n} \in D_{\omega n}} S_{\omega_{1}, \mathbf{i}_{1}} \cdots S_{\omega_{n}, \mathbf{i}_{n}}\left([0,1]^{d}\right),
$$

where the limit is with respect to the Hausdorff metric, and (1.21)

$$
\mu_{\omega}=\lim _{n} \sum_{\mathbf{i}_{1} \in D_{\omega_{1}, \ldots, \mathbf{i}_{n} \in D_{\omega_{n}}}} p_{\omega_{1}, \mathbf{i}_{1}} \cdots p_{\omega_{n}, \mathbf{i}_{n}} \frac{\left.\lambda^{d}\right|_{S_{\omega_{1}, \mathbf{i}_{1}} \cdots S_{\omega_{n}, \mathbf{i}_{n}}\left([0,1]^{d}\right)}}{\lambda^{d}\left(S_{\omega_{1}, \mathbf{i}_{1}} \cdots S_{\omega_{n}, \mathbf{i}_{n}}\left([0,1]^{d}\right)\right)},
$$

where the limit is with respect to the weak topology and

$$
\left.\lambda^{d}\right|_{S_{\omega_{1}, \mathbf{i}_{1}} \cdots S_{\omega_{n}, \mathbf{i}_{n}}\left([0,1]^{d}\right)}
$$

denotes the $d$-dimensional Lebesgue measure $\lambda^{d}$ restricted to the $d$-dimensional rectangle $S_{\omega_{1}, \mathbf{i}_{1}} \cdots S_{\omega_{n}, \mathbf{i}_{n}}\left([0,1]^{d}\right)$. Formulas (1.20) and (1.21) show that $K_{\omega}$ and $\mu_{\omega}$ can be constructed using the following recursive procedure. 
Step 1: We first select a set $D_{\omega_{1}}$ randomly from the list $\left(D_{1}, \ldots, D_{N}\right)$ according to the probability vector $\left(p_{1}, \ldots, p_{N}\right)$, and divide, in the obvious way, the $d$ dimensional unit cube $[0,1]^{d}$ into the family $\left\{S_{\omega_{1}, \mathbf{i}_{1}}\left([0,1]^{d}\right) \mid \mathbf{i}_{1} \in \Sigma_{\omega_{1}}\right\}$ of $n_{\omega_{1}, 1} \cdots n_{\omega_{1}, d}$ non-overlapping and congruent boxes with sidelengths $1 / n_{\omega_{1}, 1}, \ldots, 1 / n_{\omega_{1}, d}$. We only keep the boxes with $\mathbf{i}_{1} \in D_{\omega_{1}}$, i.e., we only keep the boxes

$$
\left\{S_{\omega_{1}, \mathbf{i}_{1}}\left([0,1]^{d}\right) \mid \mathbf{i}_{1} \in D_{\omega_{1}}\right\},
$$

and form the set

$$
K_{\left(\omega_{1}\right)}=\bigcup_{\mathbf{i}_{1} \in D_{\omega_{1}}} S_{\omega_{1}, \mathbf{i}_{1}}\left([0,1]^{d}\right) .
$$

Next, we divide a unit mass between the boxes in (1.22) in the ratio determined by the probability vector $\mathbf{p}_{\omega_{1}}$, i.e., we form the measure

$$
\mu_{\left(\omega_{1}\right)}=\sum_{\mathbf{i}_{1} \in D_{\omega_{1}}} p_{\omega_{1}, \mathbf{i}_{1}} \frac{\left.\lambda^{d}\right|_{S_{\omega_{1}, \mathbf{i}_{1}}\left([0,1]^{d}\right)}}{\lambda^{d}\left(S_{\omega_{1}, \mathbf{i}_{1}}\left([0,1]^{d}\right)\right)}
$$

such that $\mu_{\left(\omega_{1}\right)}\left(S_{\omega_{1}, \mathbf{i}_{1}}\left([0,1]^{d}\right)\right)=p_{\omega_{1}, \mathbf{i}_{1}}$ for $\mathbf{i}_{1} \in D_{\omega_{1}}$.

Step 2: Next, select a set $D_{\omega_{2}}$ randomly from the list $\left(D_{1}, \ldots, D_{N}\right)$ according to the probability vector $\left(p_{1}, \ldots, p_{N}\right)$, and divide, in the obvious way, each of the boxes $S_{\omega_{1}, \mathbf{i}_{1}}\left([0,1]^{d}\right)$ with $\mathbf{i}_{1} \in D_{\omega_{1}}$ from Step 1 into the family $\left\{S_{\omega_{1}, \mathbf{i}_{1}} S_{\omega_{2}, \mathbf{i}_{2}}\left([0,1]^{d}\right) \mid \mathbf{i}_{2} \in \Sigma_{\omega_{2}}\right\}$ of $n_{\omega_{2}, 1} \cdots n_{\omega_{2}, d}$ non-overlapping, congruent boxes with sidelengths $1 /\left(n_{\omega_{1}, 1} n_{\omega_{2}, 1}\right), \ldots, 1 /\left(n_{\omega_{1}, d} n_{\omega_{2}, d}\right)$. Again we only keep those boxes with $\mathbf{i}_{2} \in D_{\omega_{2}}$, i.e., we only keep the boxes

$$
\left\{S_{\omega_{1}, \mathbf{i}_{1}} S_{\omega_{2}, \mathbf{i}_{2}}\left([0,1]^{d}\right) \mid \mathbf{i}_{2} \in D_{\omega_{2}}\right\},
$$

and form the set

$$
K_{\left(\omega_{1}, \omega_{2}\right)}=\bigcup_{\mathbf{i}_{1} \in D_{\omega_{1}}, \mathbf{i}_{2} \in D_{\omega_{2}}} S_{\omega_{1}, \mathbf{i}_{1}} S_{\omega_{2}, \mathbf{i}_{2}}\left([0,1]^{d}\right) .
$$

Next, we divide the mass $p_{\omega_{1}, \mathbf{i}_{1}}$ of $S_{\omega_{1}, \mathbf{i}_{1}}\left([0,1]^{d}\right)$ between the boxes in (1.23) in the ratio determined by the probability vector $\mathbf{p}_{\omega_{2}}$, i.e., we form the measure

$$
\mu_{\left(\omega_{1}, \omega_{2}\right)}=\sum_{\mathbf{i}_{1} \in D \omega_{1}, \mathbf{i}_{2} \in D_{\omega_{2}}} p_{\omega_{1}, \mathbf{i}_{1}} p_{\omega_{2}, \mathbf{i}_{2}} \frac{\left.\lambda^{d}\right|_{S_{\omega_{1}, \mathbf{i}_{1}} S_{\omega_{2}, \mathbf{i}_{2}}\left([0,1]^{d}\right)}}{\lambda^{d}\left(S_{\omega_{1}, \mathbf{i}_{1}} S_{\omega_{2}, \mathbf{i}_{2}}\left([0,1]^{d}\right)\right)}
$$

such that $\mu_{\left(\omega_{1}, \omega_{2}\right)}\left(S_{\omega_{1}, \mathbf{i}_{1}} S_{\omega_{2}, \mathbf{i}_{2}}\left([0,1]^{d}\right)\right)=p_{\omega_{1}, \mathbf{i}_{1}} p_{\omega_{2}, \mathbf{i}_{2}}$ for $\mathbf{i}_{1} \in D_{\omega_{1}}$, $\mathbf{i}_{2} \in D_{\omega_{2}}$. 
By repeating this procedure, sets

$$
K_{\left(\omega_{1}, \ldots, \omega_{n}\right)}=\bigcup_{\mathbf{i}_{1} \in D_{\omega_{1}}, \ldots, \mathbf{i}_{n} \in D_{\omega_{n}}} S_{\omega_{1}, \mathbf{i}_{1}} \cdots S_{\omega_{n}, \mathbf{i}_{n}}\left([0,1]^{d}\right)
$$

and measures

$$
\mu_{\left(\omega_{1}, \ldots, \omega_{n}\right)}=\sum_{\mathbf{i}_{1} \in D_{\omega_{1}}, \ldots, \mathbf{i}_{n} \in D_{\omega_{n}}} p_{\omega_{1}, \mathbf{i}_{1}} \cdots p_{\omega_{n}, \mathbf{i}_{n}} \frac{\left.\lambda^{d}\right|_{S_{\omega_{1}, \mathbf{i}_{1}} \cdots S_{\omega_{n}, \mathbf{i}_{n}}\left([0,1]^{d}\right)}}{\lambda^{d}\left(S_{\omega_{1}, \mathbf{i}_{1}} \cdots S_{\omega_{n}, \mathbf{i}_{n}}\left([0,1]^{d}\right)\right)}
$$

are obtained. The random self-affine Sierpinski sponge $K_{\omega}$ with $\omega=\left(\omega_{1}, \omega_{2}, \ldots\right)$ is now given by

$$
K_{\omega}=\lim _{n} K_{\left(\omega_{1}, \ldots, \omega_{n}\right)}
$$

(where the limit is with respect to the Hausdorff metric), and the random multifractal self-affine Sierpinski sponge $\mu_{\omega}$ with $\omega=\left(\omega_{1}, \omega_{2}, \ldots\right)$ is given by

$$
\mu_{\omega}=\lim _{n} \mu_{\left(\omega_{1}, \ldots, \omega_{n}\right)}
$$

(where the limit is with respect to the weak topology).

We will now give an example illustrating the above construction. For this example, let $d=2, N=3$ and put $\left(n_{1,1}, n_{1,2}\right)=(4,6),\left(n_{2,1}, n_{2,2}\right)=(3,5)$ and $\left(n_{3,1}, n_{3,2}\right)=(5,8)$. Figures 1.1-1.3 illustrate the above procedure for the sets

$$
\begin{aligned}
D_{1} & =\{(1,0),(3,2),(1,4)\} \\
& \subseteq\{0,1, \ldots, 3\} \times\{0,1, \ldots, 5\}, \\
D_{2} & =\{(0,0),(2,2),(0,4),(2,4)\} \\
& \subseteq\{0,1, \ldots, 2\} \times\{0,1, \ldots, 4\}, \\
D_{3} & =\{(0,0),(2,0),(2,2),(2,4),(4,4),(0,6)\} \\
& \subseteq\{0,1, \ldots, 4\} \times\{0,1, \ldots, 7\},
\end{aligned}
$$

and the probability vectors

$$
\begin{aligned}
\mathbf{p}_{1} & =\left(p_{1,(1,0)}, p_{1,(3,2)}, p_{1,(1,4)}\right) \\
& =\left(\frac{1}{2}, \frac{1}{4}, \frac{1}{4}\right), \\
\mathbf{p}_{2} & =\left(p_{2,(0,0)}, p_{2,(2,2)}, p_{2,(0,4)}, p_{2,(2,4)}\right) \\
& =\left(\frac{1}{10}, \frac{1}{2}, \frac{3}{10}, \frac{1}{10}\right), \\
\mathbf{p}_{3} & =\left(p_{3,(0,0)}, p_{3,(2,0)}, p_{3,(2,2)}, p_{3,(2,4)}, p_{3,(4,4)}, p_{3,(0,6)}\right) \\
& =\left(\frac{1}{5}, \frac{1}{4}, \frac{1}{10}, \frac{1}{4}, \frac{1}{10}, \frac{1}{10}\right) .
\end{aligned}
$$



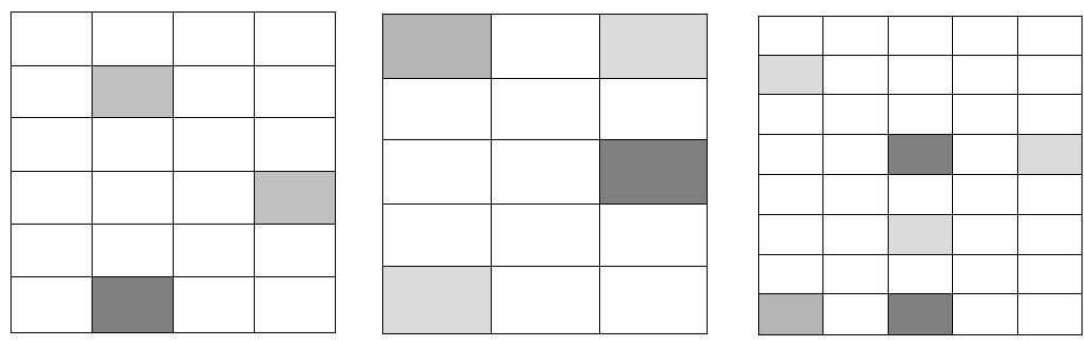

FIgURE 1.1. This figure illustrates the sets $D_{1}, D_{2}, D_{3}$ and the probability vectors $\mathbf{p}_{1}, \mathbf{p}_{2}, \mathbf{p}_{3}$ as defined above. The figure on the left shows the boxes $S_{1, \mathbf{i}}\left([0,1]^{2}\right)$ with $\mathbf{i} \in D_{1}$, and each box $S_{1, \mathbf{i}}\left([0,1]^{2}\right)$ is grey-shaded according to the probability $p_{1, \mathbf{i}}$. The figure in the middle shows the boxes $S_{2, \mathbf{i}}\left([0,1]^{2}\right)$ with $\mathbf{i} \in D_{2}$, and each box $S_{2, \mathbf{i}}\left([0,1]^{2}\right)$ is grey-shaded according to the probability $p_{2, \mathbf{i}}$. Finally, the figure on the right shows the boxes $S_{3, \mathbf{i}}\left([0,1]^{2}\right)$ with $\mathbf{i} \in D_{3}$, and each box $S_{3, \mathbf{i}}\left([0,1]^{2}\right)$ is grey-shaded according to the probability $p_{3, \mathbf{i}}$.
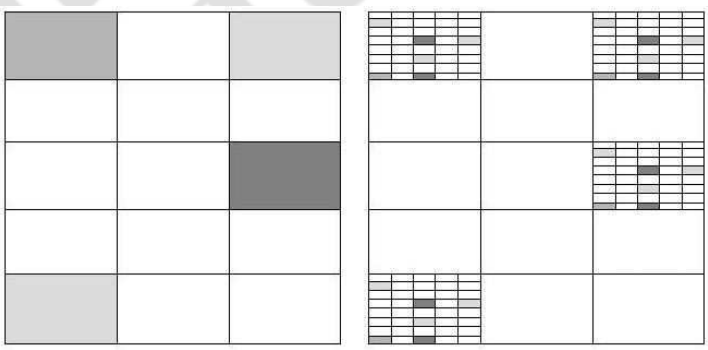

FIgURE 1.2. This figure shows the sets $K_{(2)}, K_{(2,3)}$ and the measures $\mu_{(2)}, \mu_{(2,3)}$ defined in (1.24) and (1.25) for $D_{1}, D_{2}$, $D_{3}, \mathbf{p}_{1}, \mathbf{p}_{2}, \mathbf{p}_{3}$ as defined above. The figure on the left shows $K_{(2)}$ and $\mu_{(2)}$, and the figure on the right shows $K_{(2,3)}$ and $\mu_{(2,3)}$. Each box in the figure is grey-shaded according to its mass. 

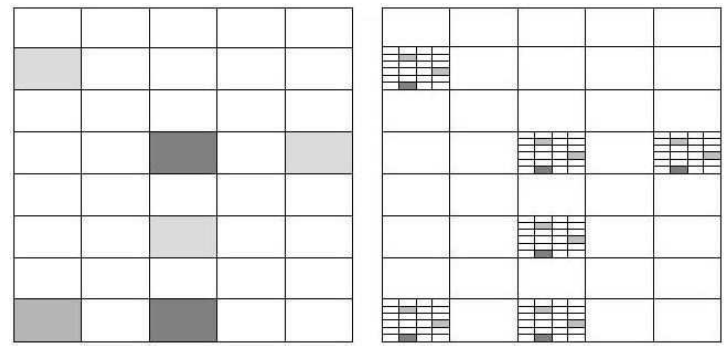

FIgURE 1.3. This figure shows the sets $K_{(3)}, K_{(3,1)}$ and the measures $\mu_{(3)}, \mu_{(3,1)}$ defined in (1.24) and (1.25) for $D_{1}, D_{2}$, $D_{3}, \mathbf{p}_{1}, \mathbf{p}_{2}, \mathbf{p}_{3}$ as defined above. The figure on the left shows $K_{(3)}$ and $\mu_{(3)}$, and the figure on the right shows $K_{(3,1)}$ and $\mu_{(3,1)}$. Each box in the figure is grey-shaded according to its mass.

\subsubsection{The Hausdorff multifractal spectrum of random self-affine multi-} fractal Sierpinski sponges. Below we compute the Hausdorff multifractal spectrum of the random self-affine measure $\mu_{\omega}$ for $\mathbb{P}$-almost all $\omega \in \Omega$. For $i=$ $1, \ldots, N$, we define auxiliary functions $b_{i, i_{1} \ldots i_{d}}, b_{i, i_{1} \ldots i_{d-1}}, \ldots, b_{i, i_{1} i_{2}}, b_{i, i_{1}}, b_{i}$ : $\mathbb{R} \rightarrow \mathbb{R}$ inductively as follows:

(0) For $q \in \mathbb{R}$ and $\left(i_{1}, \ldots, i_{d}\right) \in D_{i}$ define $b_{i, i_{1} \ldots i_{d}}(q)$ by

$$
b_{i, i_{1} \ldots i_{d}}(q)=0 \text {. }
$$

(1) For $q \in \mathbb{R}$ and $\left(i_{1}, \ldots, i_{d-1}\right) \in \pi_{d-1}\left(D_{i}\right)$ define $b_{i, i_{1} \ldots i_{d-1}}(q)$ by

$$
\sum_{\substack{i_{d} \\\left(i_{1}, \ldots, i_{d}\right) \in \pi_{d}\left(D_{i}\right)}} p_{D_{i}, \mathbf{p}_{i}, d}\left(i_{d} \mid i_{1}, \ldots, i_{d-1}\right)^{q} v_{d}^{b_{i, i_{1} \ldots i_{d}}(q)-b_{i, i_{1} \ldots i_{d-1}}(q)}=1 .
$$

(2) For $q \in \mathbb{R}$ and $\left(i_{1}, \ldots, i_{d-2}\right) \in \pi_{d-2}\left(D_{i}\right)$ define $b_{i, i_{1} \ldots i_{d-2}}(q)$ by

$$
\sum_{\substack{i_{d-1} \\\left(i_{1}, \ldots, i_{d-1}\right) \in \pi_{d-1}\left(D_{i}\right)}} p_{D_{i}, \mathbf{p}_{i}, d-1}\left(i_{d-1} \mid i_{1}, \ldots, i_{d-2}\right)^{q} v_{d-1}^{b_{i, i_{1} \ldots i_{d-1}}(q)-b_{i, i_{1} \ldots i_{d-2}}(q)}=1 .
$$

(d) For $q \in \mathbb{R}$ define $b_{i}(q)$ by

$$
\sum_{\substack{i_{1} \\ i_{1} \in \pi_{1}(D)}} p_{1}\left(i_{1}\right)^{q} v_{1}^{b_{i, i_{1}}(q)-b_{i}(q)}=1 .
$$


Hence, using the notation from Section 1.2.2, we have

$$
b_{i}=\beta_{\boldsymbol{v}, D_{i}, \mathbf{p}_{i}},
$$

i.e., $b_{i}$ is the auxiliary function defined in Section 1.2.2 associated with $D_{i}$ and $\mathbf{p}_{i}$, and the sequence $\boldsymbol{v}=\left(v_{1}, \ldots, v_{d}\right)$ of weighted multiplicative averages $v_{\ell}$ of the $n_{i, \ell}$ 's. Next, define $\beta: \mathbb{R} \rightarrow \mathbb{R}$ by

$$
\beta=\sum_{i} p_{i} b_{i}=\sum_{i} p_{i} \beta_{v, D_{i}, \mathbf{p}_{i}}
$$

and write

$$
\begin{aligned}
& \alpha_{\min , i}=\min _{\left(i_{1}, \ldots, i_{d}\right) \in D_{i}}-\sum_{\ell} \frac{\log p_{D_{i}, \mathbf{p}_{i}, \ell}\left(i_{\ell} \mid i_{1}, \ldots, i_{\ell-1}\right)}{\log n_{\ell}}, \\
& \alpha_{\max , i}=\max _{\left(i_{1}, \ldots, i_{d}\right) \in D_{i}}-\sum_{\ell} \frac{\log p_{D_{i}, \mathbf{p}_{i}, \ell}\left(i_{\ell} \mid i_{1}, \ldots, i_{\ell-1}\right)}{\log n_{\ell}} .
\end{aligned}
$$

Observe that, using the notation from Section 1.2.2, we have $\alpha_{\min , i}=\alpha_{\min , D_{i}, \mathbf{p}_{i}}$ and $\alpha_{\max , i}=\alpha_{\max , D_{i}, \mathbf{p}_{i}}$. Finally, write

$$
\begin{aligned}
& \alpha_{\min }=\sum_{i} p_{i} \alpha_{\min , i}=\sum_{i} p_{i} \alpha_{\min , D_{i}, \mathbf{p}_{i}} \\
& \alpha_{\max }=\sum_{i} p_{i} \alpha_{\max , i}=\sum_{i} p_{i} \alpha_{\max , D_{i}, \mathbf{p}_{i} .}
\end{aligned}
$$

We can now compute the Hausdorff multifractal spectrum of $\mu_{\omega}$ for $\mathbb{P}$-almost all $\omega \in \Omega$.

\section{Theorem 1.1.}

(1) For $\mathbb{P}$-almost all $\omega \in \Omega$ we have

$$
f_{\mathrm{H}, \mu_{\omega}}(\alpha)=\beta^{*}(\alpha)
$$

for all $\alpha \in\left(\alpha_{\min }, \alpha_{\max }\right)$ (here $\beta^{*}$ denotes the Legendre transform of $\beta$ defined by $\left.\beta^{*}(\alpha)=\inf _{q}(q \alpha+\beta(q))\right)$.

(2) For $\mathbb{P}$-almost all $\omega \in \Omega$ we have

$$
\left\{x \in K_{\omega} \mid \lim _{r \backslash 0} \frac{\log \mu_{\omega}(B(x, r))}{\log r}=\alpha\right\}=\emptyset
$$

for all $\alpha \notin\left[\alpha_{\min }, \alpha_{\max }\right]$.

The proof of Theorem 1.1 is given in Section 7. Observe that if we put $N=1$ and write $\mathbf{n}_{1}=\mathbf{n}, D_{1}=D$ and $\mathbf{p}_{1}=\mathbf{p}$, then Theorem 1.1 reduces to Theorem A (since in this case $\Omega$ is a singleton and $K_{\omega}=K_{\mathbf{n}, D}$ and $\mu_{\omega}=\mu_{\mathbf{n}, D, \mathbf{p}}$ for all $\omega \in \Omega)$. 
Remark. It is interesting to note that, although $\beta$ is the weighted average of the $\beta_{\boldsymbol{v}, D_{i}, \mathbf{p}_{i}}$ 's, the functions $\beta_{\boldsymbol{v}, D_{i}, \mathbf{p}_{i}}$ and their Legendre transforms $\beta_{\boldsymbol{v}, D_{i}, \mathbf{p}_{i}}^{*}$ do not have any apparent geometric significance.

Remark. In Theorem 1.1 we are assuming the VSSC. This is a very restrictive condition and it would be desirable if it could be omitted. Regrettably, we have been unable to do this. However, we are able to compute the so-called symbolic Hausdorff multifractal spectrum without assuming the VSSC. This is discussed in Section 1.3 .3 below.

Remark. Theorem 1.1 computes the Hausdorff multifractal spectrum of $\mu_{\omega}$. It is equally natural to ask for the packing multifractal spectrum of $\mu_{\omega}$ (see (1.1) for the definition of the packing multifractal spectrum of a measure). In fact, it appears that the packing multifractal spectrum is not even known for the deterministic multifractal Sierpinski sponges $\mu_{\mathbf{n}, D, \mathbf{p}}$ defined in (1.8) (however, for results related to, but different from, the packing multifractal spectrum of $\mu_{D, \mathbf{p}}$, the reader is referred to [GL5, GL6, N]).

Remark. In Theorem 1.1 we are assuming that $v_{1}<v_{2}<\cdots<v_{d}$; i.e., (see the discussion prior to (1.13)) we are assuming that the sequences $\mathbf{n}_{i}=$ $\left(n_{i, 1}, \ldots, n_{i, d}\right)$ with $i=1, \ldots, N$ are strictly increasing "on average". It would clearly be desirable if this condition could be weakened. For example, it would be desirable if we would prove Theorem 1.1 only assuming that $v_{1} \leq v_{2} \leq \cdots \leq v_{d}$. We note that strict inequalities $v_{1}<v_{2}<\cdots<v_{d}$ are used in the proof of Lemma 3.2, which plays an important role in the proofs in Section 4 and Section 5. However, despite this, we believe that the arguments in the paper can be adapted to prove Theorem 1.1 under this weaker assumption. This adaptation is outlined and discussed in Section 8.

Remark. Finally, observe that we have not been able to compute the Hausdorff spectrum $f_{\mathrm{H}, \mu_{\omega}}(\alpha)$ of $\mu_{\omega}$ for $\alpha=\alpha_{\min }$ and for $\alpha=\alpha_{\max }$.

\subsubsection{The symbolic Hausdorffmultifractal spectrum of random self-affine} multifractal Sierpinski sponges. In Theorem 1.1 we are assuming the VSSC. This is clearly a very restrictive condition, and it would be desirable if it could be omitted. Unfortunately, we have been unable to do this. However, without assuming the VSSC, we can compute the so-called symbolic Hausdorff multifractal spectrum of $\mu_{\omega}$ for $\mathbb{P}$-almost all $\omega \in \Omega$. We will now define the symbolic Hausdorff multifractal spectrum. We first define approximate cubes as follows. Fix $\omega=$ $\left(\omega_{1}, \omega_{2}, \ldots\right) \in \Omega, r>0$ and $\boldsymbol{i}=\mathbf{i}_{1} \mathbf{i}_{2} \ldots \in \Sigma_{\omega}^{\mathbb{N}}$ with $\mathbf{i}_{j}=\left(i_{j, 1}, \ldots, i_{j, d}\right) \in \Sigma_{\omega_{j}}$. We now define the approximate cube $Q_{\omega}(\boldsymbol{i}, r)$ with centre at $\boldsymbol{i}$ and diameter $r$ as follows. Namely, for each $\ell$, let $s_{\ell}$ be the unique integer such that

$$
\frac{1}{n_{\omega_{1}, \ell} n_{\omega_{2}, \ell} \ldots n_{\omega_{s_{\ell}}, \ell} n_{\omega_{s_{\ell}+1}, \ell}}<r \leq \frac{1}{n_{\omega_{1}, \ell} n_{\omega_{2}, \ell} \cdots n_{\omega_{s_{\ell}}, \ell}},
$$

and put

$$
k_{\omega, \ell}(r)=s_{\ell}
$$


Now we define $Q_{\omega}(\boldsymbol{i}, r)$ by

$$
\begin{aligned}
& Q_{\omega}(\boldsymbol{i}, r)=\underset{\ell=1}{\stackrel{d}{x}}\left[\frac{i_{1, \ell}}{n_{\omega_{1}, \ell}}+\frac{i_{2, \ell}}{n_{\omega_{1}, \ell} n_{\omega_{2}, \ell}}+\cdots+\frac{i_{k_{\omega, \ell}(r), \ell}}{n_{\omega_{1}, \ell} n_{\omega_{2}, \ell} \cdots n_{\omega_{\omega_{\omega}, \ell}(r), \ell}},\right. \\
& \frac{i_{1, \ell}}{n_{\omega_{1}, \ell}}+\frac{i_{2, \ell}}{n_{\omega_{1}, \ell} n_{\omega_{2}, \ell}}+\cdots+\frac{i_{k_{\omega, \ell}(r), \ell}}{n_{\omega_{1}, \ell} n_{\omega_{2}, \ell} \cdots n_{\omega_{k_{\omega}, \ell}(r), \ell}} \\
& \left.+\frac{1}{n_{\omega_{1}, \ell} n_{\omega_{2}, \ell} \cdots n_{\omega_{k_{\omega, \ell}(r), \ell}}}\right] \text {. }
\end{aligned}
$$

Observe that the set $Q_{\omega}(\boldsymbol{i}, r)$ is, indeed, an "approximate" cube. Namely, if we write $n_{\max }=\max _{i, \ell} n_{i, \ell}$ and $Q_{\omega}(\boldsymbol{i}, r)=\mathrm{X} \ell=1^{d} I_{\omega, \ell}(\boldsymbol{i}, r)$, then

$$
\frac{\operatorname{diam} I_{\omega, \ell_{1}}(\boldsymbol{i}, r)}{\operatorname{diam} I_{\omega, \ell_{2}}(\boldsymbol{i}, r)}=\frac{\frac{1}{n_{\omega_{1}, \ell_{1}} n_{\omega_{2}, \ell_{1}} \cdots n_{\omega_{k, \ell_{1}(r), \ell_{1}}}}}{\frac{1}{n_{\omega_{1}, \ell_{2}} n_{\omega_{2}, \ell_{2}} \cdots n_{\omega_{k_{\omega}, \ell_{2}(r), \ell_{2}}}}} \leq \frac{r n_{\max }}{r}=n_{\max },
$$

$$
\frac{\operatorname{diam} I_{\omega, \ell_{1}}(\boldsymbol{i}, r)}{\operatorname{diam} I_{\omega, \ell_{2}}(\boldsymbol{i}, r)}=\frac{\frac{1}{n_{\omega_{1}, \ell_{1}} n_{\omega_{2}, \ell_{1}} \cdots n_{\omega_{k, \ell_{1}}(r), \ell_{1}}}}{\frac{1}{n_{\omega_{1}, \ell_{2}} n_{\omega_{2}, \ell_{2}} \cdots n_{\omega_{k_{\omega, \ell}}(r), \ell_{2}}}} \geq \frac{r}{r n_{\max }}=\frac{1}{n_{\max }},
$$

for all $\ell_{1}, \ell_{2}=1, \ldots, d$, i.e., the ratio between the diameters of the intervals $I_{\omega, \ell}(\boldsymbol{i}, r)$ is bounded below and above. This shows that the set $Q_{\omega}(\boldsymbol{i}, r)=$ $\mathrm{X}_{\ell=1}^{d} I_{\omega, \ell}(\boldsymbol{i}, r)$ is, indeed, an "approximate" cube.

The symbolic Hausdorff multifractal spectrum of $\mu_{\omega}$ is now defined by

$$
f_{\mathrm{H}, \mu_{\omega}}^{\mathrm{sym}}(\alpha)=\operatorname{dim}_{\mathrm{H}} \pi_{\omega}\left\{\boldsymbol{i} \in D_{\omega}^{\mathbb{N}} \mid \lim _{r>0} \frac{\log \mu_{\omega}\left(Q_{\omega}(\boldsymbol{i}, r)\right)}{\log r}=\alpha\right\} ;
$$

i.e., we have replaced the "geometric ball" $B(x, r)$ by the "symbolic ball" $Q_{\omega}(\boldsymbol{i}, r)$. Without assuming the VSSC, we can compute the symbolic Hausdorff multifractal spectrum of $\mu_{\omega}$ for $\mathbb{P}$-almost all $\omega \in \Omega$. This is the content of the next theorem.

\section{Theorem 1.2.}

(i) For $\mathbb{P}$-almost all $\omega \in \Omega$ we have

$$
f_{\mathrm{H}, \mu_{\omega}}^{\mathrm{sym}}(\alpha)=\beta^{*}(\alpha)
$$

for all $\alpha \in\left(\alpha_{\min }, \alpha_{\max }\right)$ (here $\beta^{*}$ denotes the Legendre transform of $\beta$ defined by $\left.\beta^{*}(\alpha)=\inf _{q}(q \alpha+\beta(q))\right)$. 
(ii) For $\mathbb{P}$-almost all $\omega \in \Omega$ we have

$$
\left\{\boldsymbol{i} \in D_{\omega}^{\mathbb{N}} \mid \lim _{r \backslash 0} \frac{\log \mu_{\omega}\left(Q_{\omega}(\boldsymbol{i}, r)\right)}{\log r}=\alpha\right\}=\emptyset
$$

for all $\alpha \notin\left[\alpha_{\min }, \alpha_{\max }\right]$.

The proof of Theorem 1.2 is given in Sections 3-6. Section 3 contains various preliminary results, and Sections $4-5$ contain the main proof of Theorem 1.2. The proof of Theorem 1.2 is divided into two parts. Namely, in Section 4 we prove that for $\mathbb{P}$-almost all $\omega \in \Omega$, we have

$$
\beta^{*}(\alpha) \leq f_{\mathrm{H}, \mu_{\omega}}^{\mathrm{sym}}(\alpha) \quad \text { for all } \alpha \in\left(\alpha_{\min }, \alpha_{\max }\right),
$$

and in Section 5 we prove that for $\mathbb{P}$-almost all $\omega \in \Omega$, we have

$$
f_{\mathrm{H}, \mu_{\omega}}^{\mathrm{sym}}(\alpha) \leq \beta^{*}(\alpha) \quad \text { for all } \alpha \in\left(\alpha_{\min }, \alpha_{\max }\right),
$$

$$
\left\{\boldsymbol{i} \in D_{\omega}^{\mathbb{N}} \mid \lim _{r \backslash 0} \frac{\log \mu_{\omega}\left(Q_{\omega}(\boldsymbol{i}, r)\right)}{\log r}=\alpha\right\}=\emptyset \quad \text { for all } \alpha \notin\left[\alpha_{\min }, \alpha_{\max }\right] .
$$

Finally, in Section 6 we combine the results from Sections 4-5 to prove Theorem 1.2 .

\section{AN EXAMPLE}

We now consider a concrete example. Let $d=2, N=3$ with

$$
\begin{aligned}
& \mathbf{n}_{1}=\left(n_{1,1}, n_{1,2}\right)=(4,6), \\
& \mathbf{n}_{2}=\left(n_{2,1}, n_{2,2}\right)=(3,5), \\
& \mathbf{n}_{3}=\left(n_{3,1}, n_{3,2}\right)=(5,8)
\end{aligned}
$$

and

$$
\begin{aligned}
& D_{1}=\{(1,0),(3,2),(1,4)\}, \\
& D_{2}=\{(0,0),(2,2),(0,4),(2,4)\} \text {, } \\
& D_{3}=\{(0,0),(2,0),(2,2),(2,4),(4,4),(0,6)\} \text {, } \\
& \mathbf{p}_{1}=\left(p_{1,(1,0)}, p_{1,(3,2)}, p_{1,(0,4)}\right)=\left(\frac{1}{2}, \frac{1}{4}, \frac{1}{4}\right) \text {, } \\
& \mathbf{p}_{2}=\left(p_{2,(0,0)}, p_{2,(2,2)}, p_{2,(0,4)}, p_{2,(4,4)}\right)=\left(\frac{1}{10}, \frac{1}{2}, \frac{3}{10}, \frac{1}{10}\right) \text {, } \\
& \mathbf{p}_{3}=\left(p_{3,(0,0)}, p_{3,(2,0)}, p_{3,(2,2)}, p_{3,(2,4)}, p_{3,(4,4)}, p_{3,(0,6)}\right) \\
& =\left(\frac{1}{5}, \frac{1}{4}, \frac{1}{10}, \frac{1}{4}, \frac{1}{10}, \frac{1}{10}\right) \text {. }
\end{aligned}
$$


The sets $D_{1}, D_{2}, D_{3}$ and the probability vectors $\mathbf{p}_{1}, \mathbf{p}_{2}, \mathbf{p}_{3}$ are sketched in Figure 1.1. A straightforward calculation shows that if $\left(p_{1}, p_{2}, p_{3}\right)$ is a probability vector, then $v_{1}=4^{p_{1}} 3^{p_{2}} 5^{p_{3}}$ and $v_{2}=6^{p_{1}} 5^{p_{2}} 8^{p_{3}}$, and

$$
\begin{aligned}
& b_{1,1}(q)=\frac{\log \left((2 / 3)^{q}+(1 / 3)^{q}\right)}{\log v_{2}}, \\
& b_{1,3}(q)=0 \text {, } \\
& \beta_{\boldsymbol{v}, D_{1}, \mathbf{p}_{1}}(q)=b_{1}(q)=\frac{\log \left((3 / 4)^{q} v_{1}^{b_{1,1}(q)}+(1 / 4)^{q}\right)}{\log v_{1}}, \\
& b_{2,0}(q)=\frac{\log \left((1 / 4)^{q}+(3 / 4)^{q}\right)}{\log v_{2}}, \\
& b_{2,2}(q)=\frac{\log \left((5 / 6)^{q}+(1 / 6)^{q}\right)}{\log v_{2}}, \\
& \beta_{\boldsymbol{v}, D_{2}, \mathbf{p}_{2}}(q)=b_{2}(q)=\frac{\log \left((2 / 5)^{q} \nu_{1}^{b_{2,0}(q)}+(3 / 5)^{q} \nu_{1}^{b_{2,2}(q)}\right)}{\log \nu_{1}}, \\
& b_{3,0}(q)=\frac{\log \left((1 / 6)^{q}+(5 / 6)^{q}\right)}{\log v_{2}}, \\
& b_{3,2}(q)=\frac{\log \left(2(5 / 12)^{q}+(1 / 6)^{q}\right)}{\log v_{2}}, \\
& b_{3,4}(q)=0, \\
& \beta_{\boldsymbol{v}, D_{3}, \mathbf{p}_{3}}(q)=b_{3}(q)=\frac{\log \left((3 / 10)^{q} v_{1}^{b_{3,0}(q)}+(3 / 5)^{q} v_{1}^{b_{3,2}(q)}+(1 / 10)^{q}\right)}{\log v_{1}},
\end{aligned}
$$

for $q \in \mathbb{R}$. Finally, we have

$$
\beta(q)=p_{1} \beta_{\boldsymbol{v}, D_{1}, \mathbf{p}_{1}}(q)+p_{2} \beta_{\boldsymbol{v}, D_{2}, \mathbf{p}_{2}}(q)+p_{3} \beta_{\boldsymbol{v}, D_{3}, \mathbf{p}_{3}}(q),
$$

for $q \in \mathbb{R}$. It follows immediately from Theorem 1.1, that for $\mathbb{P}$-almost all $\omega \in \Omega$, we have

$$
f_{\mathrm{H}, \mu_{\omega}}(\alpha)=\beta^{*}(\alpha)
$$

for all $\alpha \in\left(\alpha_{\min }, \alpha_{\max }\right)$. As described in Section 1.3.1, the random measure $\mu_{\omega}$ is constructed using an inductive procedure that, at each step of the construction, randomly chooses either $\left(\mathbf{n}_{1}, D_{1}, \mathbf{p}_{1}\right),\left(\mathbf{n}_{2}, D_{2}, \mathbf{p}_{2}\right)$ or $\left(\mathbf{n}_{3}, D_{3}, \mathbf{p}_{3}\right)$ and bases that step of the construction on this choice. For this reason is it instructive to compare the almost sure Hausdorff multifractal spectrum $\beta^{*}$ of $\mu_{\omega}$ with the Hausdorff multifractal spectra of the self-affine measures $\mu_{\mathbf{n}_{i}, D_{i}, \mathbf{p}_{i}}$ from (1.8) corresponding to $\left(\mathbf{n}_{i}, D_{i}, \mathbf{p}_{i}\right)$ for $i=1,2,3$; note that it follows from Theorem A that the Hausdorff multifractal spectrum of $\mu_{\mathbf{n}_{i}, D_{i}, \mathbf{p}_{i}}$ equals the Legendre transform $\beta_{\mathbf{n}_{i}, D_{i}, \mathbf{p}_{i}}^{*}$. 
We therefore (see Figure 2.1 below) sketch the graph of function $\beta^{*}$ in (2.3) for $\left(p_{1}, p_{2}, p_{3}\right)=\left(\frac{1}{6}, \frac{1}{3}, \frac{1}{2}\right)$ and $\left(p_{1}, p_{2}, p_{3}\right)=\left(\frac{1}{10}, \frac{1}{10}, \frac{4}{5}\right)$, and the graphs of the functions $\beta_{\mathbf{n}_{1}, D_{1}, \mathbf{p}_{1}}^{*}, \beta_{\mathbf{n}_{2}, D_{2}, \mathbf{p}_{2}}^{*}$ and $\beta_{\mathbf{n}_{3}, D_{3}, \mathbf{p}_{3}}^{*}$ corresponding to the choices of $\mathbf{n}_{i}, D_{i}$ and $\mathbf{p}_{i}$ given in (2.1)-(2.2).
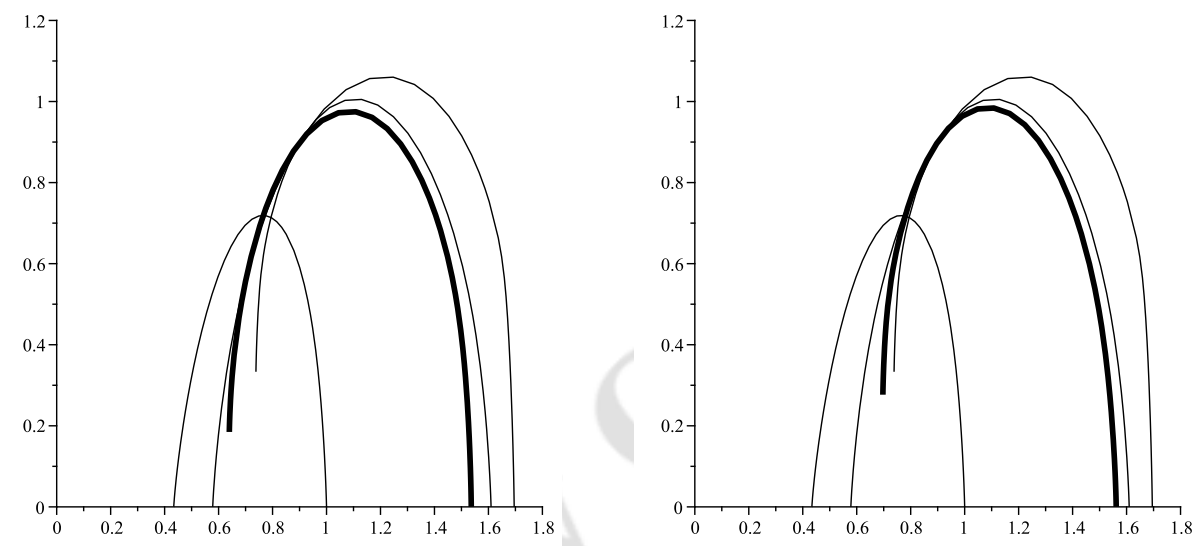

FIGURE 2.1. This figure shows the graphs of the functions $\beta_{\mathbf{n}_{1}, D_{1}, \mathbf{p}_{1}}^{*}$, $\beta_{\mathbf{n}_{2}, D_{2}, \mathbf{p}_{2}}^{*}$ and $\beta_{\mathbf{n}_{3}, D_{3}, \mathbf{p}_{3}}^{*}$ defined in Section 1.2.2 corresponding to the choices of $\mathbf{n}_{i}, D_{i}$ and $\mathbf{p}_{i}$ given in (2.1)-(2.2), and the graph of the function $\beta^{*}$ in $(2.3)$ for $\left(p_{1}, p_{2}, p_{3}\right)=\left(\frac{1}{6}, \frac{1}{3}, \frac{1}{2}\right)$ (the left figure) and $\left(p_{1}, p_{2}, p_{3}\right)=\left(\frac{1}{10}, \frac{1}{10}, \frac{4}{5}\right)$ (the right figure). The graphs of $\beta_{\mathbf{n}_{1}, D_{1}, \mathbf{p}_{1}}^{*}$, $\beta_{\mathbf{n}_{2}, D_{2}, \mathbf{p}_{2}}^{*}$ and $\beta_{\mathbf{n}_{3}, D_{3}, \mathbf{p}_{3}}^{*}$ are sketched in non-bold, and the graph of $\beta^{*}$ is sketched in bold. The graph of $\beta_{\mathbf{n}_{2}, D_{2}, \mathbf{p}_{2}}^{*}$ is the "wide" graph with the largest maximum, the graph of $\beta_{\mathbf{n}_{3}, D_{3}, \mathbf{p}_{3}}^{*}$ is the "wide" graph with the second largest maximum, and the graph of $\beta_{\mathbf{n}_{1}, D_{1}, \mathbf{p}_{1}}^{*}$ is the "narrow" graph with the smallest maximum.

\section{Proofs. Preliminary Results}

The purpose of this section is to introduce various notation and auxiliary results that are needed in Sections 4-6.

Recall, that $\Omega=\{1, \ldots, N\}^{\mathbb{N}}$. For a positive integer $n, \omega=\left(\omega_{1}, \omega_{2}, \ldots\right) \in$ $\Omega$ and $i=1, \ldots, N$, let

$$
\begin{aligned}
D_{\omega}^{n} & =\left\{\mathbf{i}_{1} \ldots \mathbf{i}_{n} \mid \mathbf{i}_{j} \in D_{\omega_{j}} \text { for all } j\right\}, \\
D_{\omega}^{\mathbb{N}} & =\left\{\mathbf{i}_{1} \mathbf{i}_{2} \ldots \mid \mathbf{i}_{j} \in D_{\omega_{j}} \text { for all } j\right\}, \\
D_{i}^{n} & =\left\{\mathbf{i}_{1} \ldots \mathbf{i}_{n} \mid \mathbf{i}_{j} \in D_{i} \text { for all } j\right\}, \\
D_{i}^{\mathbb{N}} & =\left\{\mathbf{i}_{1} \mathbf{i}_{2} \ldots \mid \mathbf{i}_{j} \in D_{i} \text { for all } j\right\} ;
\end{aligned}
$$


i.e., $D_{\omega}^{n}$ denotes the family of all finite strings $\boldsymbol{i}=\mathbf{i}_{1} \ldots \mathbf{i}_{n}$ of length $n$ with entries $\mathbf{i}_{j} \in D_{\omega_{j}}$ for all $j, D_{\omega}^{\mathbb{N}}$ denotes the family of all infinite strings $\boldsymbol{i}=\mathbf{i}_{1} \mathbf{i}_{2} \ldots$ with entries $\mathbf{i}_{j} \in D_{\omega_{j}}$ for all $j, D_{i}^{n}$ denotes the family of all finite strings $\boldsymbol{i}=\mathbf{i}_{1} \ldots \mathbf{i}_{n}$ of length $n$ with entries $\mathbf{i}_{j} \in D_{i}$ for all $j$ and $D_{i}^{\mathbb{N}}$ denotes the family of all infinite strings $\boldsymbol{i}=\mathbf{i}_{1} \mathbf{i}_{2} \ldots$ with entries $\mathbf{i}_{j} \in D_{i}$ for all $j$.

We define cylinders in $D_{\omega}^{\mathbb{N}}$ and $D_{i}^{\mathbb{N}}$ as follows. For each positive integer $n$ and $\mathbf{i}_{1} \ldots \mathbf{i}_{n} \in D_{\omega}^{n}$, we define the cylinder $\left[\mathbf{i}_{1} \ldots \mathbf{i}_{n}\right]$ in $D_{\omega}^{\mathbb{N}}$ generated by the finite string $\mathbf{i}_{1} \ldots \mathbf{i}_{n}$, by

$$
\left[\mathbf{i}_{1} \ldots \mathbf{i}_{n}\right]=\left\{\mathbf{j}_{1} \mathbf{j}_{2} \ldots \in D_{\omega}^{\mathbb{N}} \mid \mathbf{j}_{1}=\mathbf{i}_{1}, \ldots, \mathbf{j}_{n}=\mathbf{i}_{n}\right\} .
$$

In addition, for each positive integer $n$ and $\mathbf{i}_{1} \ldots \mathbf{i}_{n} \in D_{i}^{n}$, we define the cylinder $\left[\mathbf{i}_{1} \ldots \mathbf{i}_{n}\right]$ in $D_{i}^{\mathbb{N}}$ generated by the finite string $\mathbf{i}_{1} \ldots \mathbf{i}_{n}$, by

$$
\left[\mathbf{i}_{1} \ldots \mathbf{i}_{n}\right]=\left\{\mathbf{j}_{1} \mathbf{j}_{2} \ldots \in D_{i}^{\mathbb{N}} \mid \mathbf{j}_{1}=\mathbf{i}_{1}, \ldots, \mathbf{j}_{n}=\mathbf{i}_{n}\right\}
$$

3.1. Approximate cubes. We now present two useful lemmas describing the asymptotic geometry of approximate cubes. However, for the benefit of the reader we first recall the definition of an approximate cube. Fix $\omega=\left(\omega_{1}, \omega_{2}, \ldots\right) \in \Omega$, $r>0$ and $\boldsymbol{i}=\mathbf{i}_{1} \mathbf{i}_{2} \ldots \in \Sigma_{\omega}^{\mathbb{N}}$ with $\mathbf{i}_{j}=\left(i_{j, 1}, \ldots, i_{j, d}\right) \in \Sigma_{\omega_{j}}$. We now define the approximate cube $Q_{\omega}(\boldsymbol{i}, r)$ with centre at $\boldsymbol{i}$ and diameter $r$ as follows. Namely, for each $\ell$, let $s_{\ell}$ be the unique integer such that

$$
\frac{1}{n_{\omega_{1}, \ell} n_{\omega_{2}, \ell} \cdots n_{\omega_{s_{\ell}}, \ell} n_{\omega_{s_{\ell}+1}, \ell}}<r \leq \frac{1}{n_{\omega_{1}, \ell} n_{\omega_{2}, \ell} \cdots n_{\omega_{s_{\ell}}, \ell}},
$$

and put

$$
k_{\omega, \ell}(r)=s_{\ell}
$$

Now we define $Q_{\omega}(\boldsymbol{i}, r)$ by

$$
\begin{aligned}
& Q_{\omega}(\boldsymbol{i}, r)=\stackrel{d}{x}\left[\frac{i_{1, \ell}}{n_{\omega_{1}, \ell}}+\frac{i_{2, \ell}}{n_{\omega_{1}, \ell} n_{\omega_{2}, \ell}}+\cdots+\frac{i_{k_{\omega, \ell}(r), \ell}}{n_{\omega_{1}, \ell} n_{\omega_{2}, \ell} \cdots n_{\omega_{\omega_{\omega}, \ell}(r), \ell}},\right. \\
& \frac{i_{1, \ell}}{n_{\omega_{1}, \ell}}+\frac{i_{2, \ell}}{n_{\omega_{1}, \ell} n_{\omega_{2}, \ell}}+\cdots+\frac{i_{k_{\omega, \ell}(r), \ell}}{n_{\omega_{1}, \ell} n_{\omega_{2}, \ell} \cdots n_{\omega_{k_{\omega, \ell}(r), \ell}}} \\
& \left.+\frac{1}{n_{\omega_{1}, \ell} n_{\omega_{2}, \ell} \cdots n_{\omega_{k_{\omega, \ell}(r), \ell}}}\right] \text {. }
\end{aligned}
$$

Next, we present two lemmas describing the asymptotic behaviour of $k_{\omega, \ell}(r)$ for $\mathbb{P}$-almost all $\omega \in \Omega$ as $r \backslash 0$. 
Lemma 3.1. For $\mathbb{P}$-almost all $\omega \in \Omega$, we have

$$
\lim _{r \backslash 0} \frac{\log r}{k_{\omega, \ell}(r)}=-\sum_{i} p_{i} \log n_{i, \ell}=-\log v_{\ell}
$$

Proof. For each $\omega=\left(\omega_{1}, \omega_{2}, \ldots\right) \in \Omega$, it follows from the definition of $k_{\omega, \ell}(r)$ that

$$
-\frac{1}{k_{\omega, \ell}(r)} \sum_{j=1}^{k_{\omega, \ell}(r)} \log n_{\omega_{j}, \ell} \leq \frac{\log r}{k_{\omega, \ell}(r)} \leq-\frac{1}{k_{\omega, \ell}(r)} \sum_{j=1}^{k_{\omega, \ell}(r)+1} \log n_{\omega_{j}, \ell} .
$$

The desired result follows immediately from the above inequalities and the ergodic theorem.

Lemma 3.2. There is a set $V \subseteq \Omega$ such that

(1) $\mathbb{P}(V)=1$;

(2) for all $\omega \in V$ there is a real number $r_{0}(\omega)>0$ such that $k_{\omega, 1}(r) \geq$ $k_{\omega, 2}(r) \geq \cdots \geq k_{\omega, d}(r)$ for all $0<r<r_{0}(\omega)$.

Proof. Since $v_{1}<v_{2}<\cdots<v_{d}$ (see (1.14)), it follows from Lemma 3.1 that $k_{\omega, \ell+1}(r) / k_{\omega, \ell}(r) \rightarrow \log v_{\ell} / \log v_{\ell+1}<1$ for $\mathbb{P}$-almost all $\omega \in \Omega$ and all $\ell$. This completes the proof.

3.2. The auxiliary measures $\tilde{\mu}_{\omega}$ and $\tilde{\mu}_{i}$. Let $\omega=\left(\omega_{1}, \omega_{2}, \ldots\right) \in \Omega$ and $i=1, \ldots, N$. Since $\left(p_{i, \mathbf{i}}\right)_{\mathbf{i} \in D_{i}}$ is a probability vector, we can define measures $\tilde{\mu}_{\omega}$ and $\tilde{\mu}_{i}$ on $D_{\omega}^{\mathbb{N}}$ and $D_{i}^{\mathbb{N}}$, respectively, by

$$
\tilde{\mu}_{\omega}\left(\left[\mathbf{i}_{1} \ldots \mathbf{i}_{n}\right]\right)=p_{\omega_{1}, \mathbf{i}_{1}} \cdots p_{\omega_{n}, \mathbf{i}_{n}}
$$

for all positive integers $n$ and all $\mathbf{i}_{j} \in D_{\omega_{j}}$, and

$$
\tilde{\mu}_{i}\left(\left[\mathbf{i}_{1} \ldots \mathbf{i}_{n}\right]\right)=p_{i, \mathbf{i}_{1}} \cdots p_{i, \mathbf{i}_{n}}
$$

for all positive integers $n$ and all $\mathbf{i}_{j} \in D_{i}$. Next recall that the measure $\mu_{\omega}$ is the unique probability measure on $K_{\omega}$ such that

$$
\mu_{\omega}\left(S_{\omega_{1}, \mathbf{i}_{1}} \cdots S_{\omega_{n}, \mathbf{i}_{n}}\left([0,1]^{d}\right)\right)=p_{\omega_{1}, \mathbf{i}_{1}} \cdots p_{\omega_{n}, \mathbf{i}_{n}}
$$

for all positive integers $n$ and all strings $\mathbf{i}_{1} \ldots \mathbf{i}_{n}$ with $\mathbf{i}_{j} \in D_{\omega_{j}}$.

Fix $i=1, \ldots, N$. Also, recall that for $\ell=0,1, \ldots, d$ and $\left(i_{1}, \ldots, i_{\ell}\right) \in$ $\pi_{\ell}\left(D_{i}\right)$, we define conditional probabilities $p_{D_{i}, \mathbf{p}_{i}, \ell}\left(i_{\ell} \mid i_{1}, \ldots, i_{\ell-1}\right)$ in (1.10). 
For brevity, we will write $p_{i, \ell}\left(i_{\ell} \mid i_{1}, \ldots, i_{\ell-1}\right)=p_{D_{i}, \mathbf{p}_{i}, \ell}\left(i_{\ell} \mid i_{1}, \ldots, i_{\ell-1}\right)$; i.e., we write

$$
p_{i, \ell}\left(i_{\ell} \mid i_{1}, \ldots, i_{\ell-1}\right)=\frac{\sum_{\substack{\mathbf{j}=\left(j_{1}, \ldots, j_{d}\right) \in D_{i} \\ j_{1}=i_{1}, \ldots, j_{\ell}=i_{\ell}}} p_{i, \mathbf{j}}}{\sum_{\substack{\mathbf{j}=\left(j_{1}, \ldots, j_{d}\right) \in D_{i} \\ j_{1}=i_{1}, \ldots, j_{\ell-1}=i_{\ell-1}}} p_{i, \mathbf{j}}} .
$$

Also write

$$
q_{i, \ell}\left(i_{1}, \ldots, i_{\ell}\right)=p_{i, 1}\left(i_{1}\right) p_{i, 2}\left(i_{2} \mid i_{1}\right) \cdots p_{i, \ell}\left(i_{\ell} \mid i_{1}, \ldots, i_{\ell-1}\right) .
$$

Finally, we compute the $\mu_{\omega}$ measure of an approximate cube.

Lemma 3.3. Let $\omega=\left(\omega_{1}, \omega_{2}, \ldots\right) \in V$ (where $V$ is defined in Lemma 3.2). For $\boldsymbol{i}=\mathbf{i}_{1} \mathbf{i}_{2} \ldots \in D_{\omega}^{\mathbb{N}}$ with $\mathbf{i}_{j}=\left(i_{j, 1}, \ldots, i_{j, d}\right) \in D_{\omega_{j}}$ and $0<r<r_{0}(\omega)$ (where $r_{0}(\omega)$ is defined in Lemma 3.2), we have

$$
\mu_{\omega}\left(Q_{\omega}(\boldsymbol{i}, r)\right)=\prod_{\ell=1}^{d} \prod_{j=1}^{k_{\omega, \ell}(r)} p_{\omega_{j}, \ell}\left(i_{j, \ell} \mid i_{j, 1}, \ldots, i_{j, \ell-1}\right) .
$$

Proof. This follows immediately from the definition of $\mu_{\omega}$ and the fact that if $\omega \in V$, then $k_{\omega, 1}(r) \geq k_{\omega, 2}(r) \geq \cdots \geq k_{\omega, d}(r)$ for $0<r<r_{0}(\omega)$.

3.3. The auxiliary measures $\tilde{\mu}_{\omega}^{q}$ and $\tilde{\mu}_{i}^{q}$. Fix $q \in \mathbb{R}$. Let

$$
P_{i, \mathbf{i}}(q)=p_{i, \mathbf{i}}^{q} v_{d}^{b_{i, i_{1} \ldots i_{d}}(q)-b_{i, i_{1} \ldots i_{d-1}}(q)} v_{d-1}^{b_{i, i_{1} \ldots i_{d-1}}(q)-b_{i, i_{1} \ldots i_{d-2}}(q)} \cdots v_{1}^{b_{i, i_{1}}(q)-b_{i}(q)}
$$

Let $\omega=\left(\omega_{1}, \omega_{2}, \ldots\right) \in \Omega$ and $i=1, \ldots, N$. Since it is not difficult to see that $\left(P_{i, \mathbf{i}}(q)\right)_{\mathbf{i} \in D_{i}}$ is a probability vector, we can define measures $\tilde{\mu}_{\omega}^{q}$ and $\tilde{\mu}_{i}^{q}$ on $D_{\omega}^{\mathbb{N}}$ and $D_{i}^{\mathbb{N}}$, respectively, by

$$
\tilde{\mu}_{\omega}^{q}\left(\left[\mathbf{i}_{1} \ldots \mathbf{i}_{n}\right]\right)=P_{\omega_{1}, \mathbf{i}_{1}}(q) \cdots P_{\omega_{n}, \mathbf{i}_{n}}(q)
$$

for all positive integers $n$ and all $\mathbf{i}_{j} \in D_{\omega_{j}}$, and

$$
\tilde{\mu}_{i}^{q}\left(\left[\mathbf{i}_{1} \ldots \mathbf{i}_{n}\right]\right)=P_{i, \mathbf{i}_{1}}(q) \cdots P_{i, \mathbf{i}_{n}}(q)
$$

for all positive integers $n$ and all $\mathbf{i}_{j} \in D_{i}$. Next let $\mu_{\omega}^{q}$ be the unique probability measure on $K_{\omega}$ such that

$$
\mu_{\omega}^{q}\left(S_{\omega_{1}, \mathbf{i}_{1}} \cdots S_{\omega_{n}, \mathbf{i}_{n}}\left([0,1]^{d}\right)\right)=P_{\omega_{1}, \mathbf{i}_{1}}(q) \cdots P_{\omega_{n}, \mathbf{i}_{n}}(q)
$$


for all positive integers $n$ and all strings $\mathbf{i}_{1} \ldots \mathbf{i}_{n}$ with $\mathbf{i}_{j} \in D_{\omega_{j}}$.

Fix $i=1, \ldots, N$. For $\ell=0,1, \ldots, d$ and $\left(i_{1}, \ldots, i_{\ell}\right) \in \pi_{\ell}\left(D_{i}\right)$, we define conditional probabilities $P_{i, \ell}\left(q ; i_{\ell} \mid i_{1}, \ldots, i_{\ell-1}\right)$ by

$$
P_{i, \ell}\left(q ; i_{\ell} \mid i_{1}, \ldots, i_{\ell-1}\right)=\frac{\sum_{\substack{\mathbf{j}=\left(j_{1}, \ldots, j_{d}\right) \in D_{i} \\ j_{1}=i_{1}, \ldots, j_{\ell}=i_{\ell}}} P_{i, \mathbf{j}}(q)}{\sum_{\substack{\mathbf{j}=\left(j_{1}, \ldots, j_{d}\right) \in D_{i} \\ j_{1}=i_{1}, \ldots, j_{\ell-1}=i_{\ell-1}}} P_{i, \mathbf{j}}(q)} .
$$

Also write

$$
Q_{i, \ell}\left(q ; i_{1}, \ldots, i_{\ell}\right)=P_{i, 1}\left(q ; i_{1}\right) P_{i, 2}\left(q ; i_{2} \mid i_{1}\right) \cdots P_{i, \ell}\left(q ; i_{\ell} \mid i_{1}, \ldots, i_{\ell-1}\right) .
$$

Finally, we compute the $\mu_{\omega}^{q}$ measure of an approximate cube.

Lemma 3.4. Let $\omega=\left(\omega_{1}, \omega_{2}, \ldots\right) \in V$ (where $V$ is defined in Lemma 3.2). Fix $q \in \mathbb{R}$. For $\boldsymbol{i}=\mathbf{i}_{1} \mathbf{i}_{2} \ldots \in D_{\omega}^{\mathbb{N}}$ with $\mathbf{i}_{j}=\left(i_{j, 1}, \ldots, i_{j, d}\right) \in D_{\omega_{j}}$ and $0<r<$ $r_{0}(\omega)$ (where $r_{0}(\omega)$ is defined in Lemma 3.2), we have

$$
\mu_{\omega}^{q}\left(Q_{\omega}(\boldsymbol{i}, r)\right)=\prod_{\ell=1}^{d} \prod_{j=1}^{k_{\omega, \ell}(r)} P_{\omega_{j}, \ell}\left(q ; i_{j, \ell} \mid i_{j, 1}, \ldots, i_{j, \ell-1}\right) .
$$

Proof. This follows immediately from the definition of $\mu_{\omega}^{q}$ and the fact that if $\omega \in V$, then $k_{\omega, 1}(r) \geq k_{\omega, 2}(r) \geq \cdots \geq k_{\omega, d}(r)$ for $0<r<r_{0}(\omega)$.

3.4. A useful lemma. We close this section with a useful lemma. This lemma will be used throughout the remaining sections without being mentioned explicitly.

Lemma 3.5. Fix $q \in \mathbb{R}$ and $i=1, \ldots, N$. Let $\left(i_{1}, \ldots, i_{\ell}\right) \in \pi_{\ell}\left(D_{i}\right)$. Then

$$
\frac{Q_{i, \ell}\left(q ; i_{1}, \ldots, i_{\ell}\right)}{q_{i, \ell}\left(i_{1}, \ldots, i_{\ell}\right)^{q}}=v_{\ell}^{b_{i, i_{1} \ldots i_{\ell}}(q)-b_{i, i_{1} \ldots i_{\ell-1}}(q)} \ldots v_{2}^{b_{i, i_{1} i_{2}}(q)-b_{i, i_{1}}(q)} v_{1}^{b_{i, i_{1}}(q)-b_{i}(q)}
$$

Proof. This follows immediately from the definitions.

\section{Proof of The Lower Bound (1.33) In Theorem 1.2}

The purpose of this section is to prove the lower bound (1.33) in Theorem 1.2.

We begin with two small lemmas providing explicit expressions for $-\beta^{\prime}(q)$ and $-q \beta^{\prime}(q)+\beta(q)$, and for the range of $-\beta^{\prime}$, respectively. 
Lemma 4.1. Fix $q \in \mathbb{R}$ and $i=1, \ldots, N$.

(1) We have

(4.1) $\begin{aligned}-\beta^{\prime}(q)=-\sum_{i=1}^{N} p_{i} \sum_{\ell=1}^{d} \frac{1}{\log v_{\ell}} \sum_{\left(i_{1}, \ldots, i_{\ell}\right) \in \pi_{\ell}\left(D_{i}\right)} Q_{i, \ell}\left(q ; i_{1}, \ldots, i_{\ell}\right) \\ \times \log p_{i, \ell}\left(i_{\ell} \mid i_{1}, \ldots, i_{\ell-1}\right) .\end{aligned}$

(2) We have

$-q \beta^{\prime}(q)+\beta(q)=\sum_{i=1}^{N} p_{i} \sum_{\ell=1}^{d} \frac{1}{\log v_{\ell}} \sum_{\left(i_{1}, \ldots, i_{\ell}\right) \in \pi_{\ell}\left(D_{i}\right)} Q_{i, \ell}\left(q ; i_{1}, \ldots, i_{\ell}\right)$

$\times \log P_{i, \ell}\left(q ; i_{\ell} \mid i_{1}, \ldots, i_{\ell-1}\right)$.

Proof. (1) The definitions of $b_{i}, b_{i, i_{1}}, b_{i, i_{1} i_{2}}, \ldots, b_{i, i_{1} \ldots i_{d}}$ imply that

$$
\begin{aligned}
& -b_{i, i_{1} \ldots i_{\ell-1}}^{\prime}(q)=-\frac{1}{\log v_{\ell}} \quad \sum_{i_{\ell}} p_{i, \ell}\left(i_{\ell} \mid i_{1}, \ldots, i_{\ell-1}\right)^{q} v_{\ell}^{b_{i, i_{1} \ldots i_{\ell}}(q)-b_{i, i_{1} \ldots i_{\ell-1}}(q)} \\
& \left(i_{1}, \ldots, i_{\ell}\right) \in \pi_{\ell}\left(D_{i}\right) \times \log p_{i, \ell}\left(i_{\ell} \mid i_{1} \ldots i_{\ell-1}\right) \\
& -\sum_{i_{\ell}} p_{i, \ell}\left(i_{\ell} \mid i_{1}, \ldots, i_{\ell-1}\right)^{q} v_{\ell}^{b_{i, i_{1} \ldots i_{\ell}}(q)-b_{i, i_{1} \ldots i_{\ell-1}}(q)} b_{i_{1} \ldots i_{\ell}}^{\prime}(q) \\
& \left(i_{1}, \ldots, i_{\ell}\right) \in \pi_{\ell}\left(D_{i}\right)
\end{aligned}
$$

for $\ell=1, \ldots, d$. It follows from repeated application of (4.3), Lemma 3.5 and the fact that $b_{i, i_{1} \ldots i_{d}}(q)=0$ for all $q$ that

$$
\begin{aligned}
&-b_{i}^{\prime}(q)=- \sum_{\ell=1}^{d} \frac{1}{\log v_{\ell}} \sum_{\substack{i_{1} \\
i_{1} \in \pi_{1}\left(D_{i}\right)}} \sum_{\substack{i_{2} \\
\left(i_{1}, i_{2}\right) \in \pi_{2}\left(D_{i}\right)}} \ldots \sum_{\substack{i_{\ell} \\
\left(i_{1}, \ldots, i_{\ell}\right) \in \pi_{\ell}\left(D_{i}\right)}} q_{i, \ell}\left(i_{1}, \ldots, i_{\ell}\right)^{q} \\
& \times v_{\ell}^{b_{i, i_{1} \ldots i_{\ell}}(q)-b_{i, i_{1} \ldots i_{\ell-1}}(q)} \ldots v_{2}^{b_{i, i_{1} i_{2}}(q)-b_{i, i_{1}}(q)} v_{1}^{b_{i, i_{1}}(q)-b_{i}(q)} \\
& \quad \times \log p_{i, \ell}\left(i_{\ell} \mid i_{1}, \ldots, i_{\ell-1}\right) \\
&=-\sum_{\ell=1}^{d} \frac{1}{\log v_{\ell}} \sum_{\left(i_{1}, \ldots, i_{\ell}\right) \in \pi_{\ell}\left(D_{i}\right)} Q_{i, \ell}\left(q ; i_{1}, \ldots, i_{\ell}\right) \log p_{i, \ell}\left(i_{\ell} \mid i_{1}, \ldots, i_{\ell-1}\right) .
\end{aligned}
$$

Finally, since $\beta=\sum_{i} p_{i} b_{i}$, the desired result follows from (4.4). 
(2) For each $\ell=1, \ldots, d$, we deduce from Lemma 3.5 that

(4.5) $\log P_{i, \ell}\left(q ; i_{\ell} \mid i_{1}, \ldots, i_{\ell-1}\right)$

$$
\begin{aligned}
& =\log \frac{Q_{i, \ell}\left(q ; i_{1}, \ldots, i_{\ell}\right)}{Q_{i, \ell}\left(q ; i_{1}, \ldots, i_{\ell-1}\right)} \\
& =\log \left(\left(\frac{q_{i, \ell}\left(i_{1}, \ldots, i_{\ell}\right)}{q_{i, \ell}\left(i_{1}, \ldots, i_{\ell-1}\right)}\right)^{q} v_{\ell}^{b_{i_{1} \ldots i_{\ell}}(q)-b_{i_{1} \ldots i_{\ell-1}}(q)}\right) \\
& =q \log p_{i, \ell}\left(i_{\ell} \mid i_{1}, \ldots, i_{\ell-1}\right)+\left(b_{i, i_{1} \ldots i_{\ell}}(q)-b_{i, i_{1} \ldots i_{\ell-1}}(q)\right) \log v_{\ell} .
\end{aligned}
$$

For brevity, we now write the right-hand side of equation (4.2) as $\sum_{i=1}^{N} p_{i} \Phi_{i}$, i.e., we put

$$
\Phi_{i}=\sum_{\ell=1}^{d} \frac{1}{\log v_{\ell}} \sum_{\left(i_{1}, \ldots, i_{\ell}\right) \in \pi_{\ell}\left(D_{i}\right)} Q_{i, \ell}\left(q ; i_{1}, \ldots, i_{\ell}\right) \log P_{i, \ell}\left(q ; i_{\ell} \mid i_{1}, \ldots, i_{\ell-1}\right) .
$$

It follows from Part (1) of the lemma (i.e., (4.1)) and (4.5) that

$$
\begin{aligned}
& \Phi_{i}=-q \sum_{\ell=1}^{d}\left(\frac{1}{\log v_{\ell}} \sum_{\left(i_{1}, \ldots, i_{\ell}\right) \in \pi_{\ell}\left(D_{i}\right)} Q_{i, \ell}\left(q ; i_{1}, \ldots, i_{\ell}\right) \log p_{i, \ell}\left(i_{\ell} \mid i_{1}, \ldots, i_{\ell-1}\right)\right) \\
&-\sum_{\ell=1}^{d}\left(\frac{1}{\log v_{\ell}} \sum_{\left(i_{1}, \ldots, i_{\ell}\right) \in \pi_{\ell}\left(D_{i}\right)} Q_{i, \ell}\left(q ; i_{1}, \ldots, i_{\ell}\right)\right. \\
& \times\left.\left.-q b_{i}^{\prime}(q)-\sum_{\ell, i_{1} \ldots i_{\ell}}(q)-b_{i, i_{1} \ldots i_{\ell-1}}(q)\right) \log v_{\ell}\right) \\
&=\left.\left.-q b_{i}^{\prime}(q)+\sum_{i_{1} \in \pi_{1}\left(D_{i}\right)}^{d} Q_{\left.i_{1}, \ldots, i_{\ell}\right) \in \pi_{\ell}\left(D_{i}\right)} Q_{i, \ell}\left(q ; i_{1}\right) b_{i}(q) i_{1}, \ldots, i_{\ell}\right)\left(b_{i, i_{1} \ldots i_{\ell}}(q)-b_{i, i_{1} \ldots i_{\ell-1}}(q)\right)\right) \\
&-\sum_{\ell=1}^{d-1}\left(\sum_{\left(i_{1}, \ldots, i_{\ell}\right) \in \pi_{i}\left(D_{\ell}\right)} Q_{i, \ell}\left(q ; i_{1}, \ldots, i_{\ell}\right) b_{i, i_{1} \ldots i_{\ell}}(q)\right) \\
&+\sum_{\ell=1}^{d-1}\left(\sum_{\left(i_{1}, \ldots, i_{\ell}\right) \in \pi_{i}\left(D_{\ell}\right)} Q_{i, \ell}\left(q ; i_{1}, \ldots, i_{\ell}\right) b_{i, i_{1} \ldots i_{\ell}}(q)\right. \\
&=-q b_{i}^{\prime}(q)+b_{i}(q) . \\
&\left.\sum_{i_{\ell+1}} P_{i, \ell+1}\left(q ; i_{\ell+1} \mid i_{1}, \ldots, i_{\ell}\right)\right) \\
&\left(i_{1}, \ldots, i_{\ell+1}\right) \in \pi_{\ell+1}\left(D_{i}\right)
\end{aligned}
$$


As in Part (1) of the lemma, since $\beta=\sum_{i} p_{i} b_{i}$, the desired result follows from (4.6).

\section{Lemma 4.2.}

(1) The function $-\beta^{\prime}$ is continuous (even real analytic) and decreasing with

$$
\lim _{q \rightarrow-\infty}-\beta^{\prime}(q)=\alpha_{\max }, \quad \lim _{q \rightarrow \infty}-\beta^{\prime}(q)=\alpha_{\min }
$$

(recall that the numbers $\alpha_{\min }$ and $\alpha_{\max }$ are defined in (1.29)).

(2) We have $\left\{-\beta^{\prime}(q) \mid q \in \mathbb{R}\right\}^{\circ}=\left(\alpha_{\min }, \alpha_{\max }\right)$.

(3) If $\alpha \notin\left[\alpha_{\min }, \alpha_{\max }\right]$, then $\beta^{*}(\alpha)=-\infty$.

(4) If $\alpha \in\left(\alpha_{\min }, \alpha_{\max }\right)$, then $\beta^{*}(\alpha)>0$.

Proof. (1) Since $b_{i}=\beta_{\boldsymbol{v}, D_{i}, \mathbf{p}_{i}}$ (cf. (1.26)), arguments similar to those in $[\mathrm{CM}]$ or [O1], but rather more elaborate, show that $-b_{i}^{\prime}$ is continuous (even real analytic) and decreasing with $\lim _{q \rightarrow-\infty}-b_{i}^{\prime}(q)=\alpha_{\max , i}$ and $\lim _{q \rightarrow \infty}-b_{i}^{\prime}(q)=$ $\alpha_{\min , i}$ (recall that the numbers $\alpha_{\min , i}$ and $\alpha_{\max , i}$ are defined in (1.28)). Since $\beta=\sum_{i} p_{i} b_{i}$, we therefore conclude that $-\beta^{\prime}=\sum_{i}-p_{i} b_{i}^{\prime}$ is continuous and decreasing with $\lim _{q \rightarrow-\infty}-\beta^{\prime}(q)=\sum_{i} p_{i}\left(\lim _{q \rightarrow-\infty}-b_{i}^{\prime}(q)\right)=\sum_{i} p_{i} \alpha_{\max , i}=\alpha_{\max }$ and $\lim _{q \rightarrow \infty}-\beta^{\prime}(q)=\sum_{i} p_{i}\left(\lim _{q \rightarrow \infty}-b_{i}^{\prime}(q)\right)=\sum_{i} p_{i} \alpha_{\min , i}=\alpha_{\min }$.

(2)-(4) The statements in (2)-(4) follow easily from Part (1) using standard results from convex analysis, see, for example, [R3].

Next, we introduce some notation that will be used in Lemma 4.3 and Proposition 4.4. Let

$$
U=\left\{\left(\omega_{1}, \omega_{2}, \ldots\right) \in \Omega:\left|\left\{j \mid \omega_{j}=i\right\}\right|=\infty \text { for all } i=1, \ldots, N\right\} .
$$

It is easily seen that

$$
\mathbb{P}(U)=1 .
$$

Indeed, writing $I=\{1, \ldots, N\}$ and $I_{i}=\{1, \ldots, N\} \backslash\{i\}$, we clearly have $\Omega \backslash U=$ $\bigcup_{i} \bigcup_{n}\left(I^{n} \times I_{i}^{\mathbb{N}}\right)$, whence $\mathbb{P}(\Omega \backslash U) \leq \sum_{i} \sum_{n} \mathbb{P}\left(I^{n} \times I_{i}^{\mathbb{N}}\right)=0$ where the last equality is due to the fact that $\mathbb{P}\left(I^{n} \times I_{i}^{\mathbb{N}}\right)=0$. This proves that $\mathbb{P}(U)=1$. by

Also, for $\omega=\left(\omega_{1}, \omega_{2}, \ldots\right) \in U$ and $i=1, \ldots, N$, define $\Pi_{\omega, i}: D_{\omega}^{\mathbb{N}} \rightarrow D_{i}^{\mathbb{N}}$

$$
\Pi_{\omega, i}\left(\mathbf{i}_{1} \mathbf{i}_{2} \ldots\right)=\mathbf{i}_{j_{1}} \mathbf{i}_{j_{2}} \ldots,
$$

where $j_{1}<j_{2}<\cdots$ and $\left\{j_{1}, j_{2}, \ldots\right\}=\left\{j \mid \omega_{j}=i\right\}$ (observe that since $\omega=\left(\omega_{1}, \omega_{2}, \ldots\right) \in U$, the set $\left\{j_{1}, j_{2}, \ldots\right\}=\left\{j \mid \omega_{j}=i\right\}$ is infinite, and, consequently, $\Pi_{\omega, i}$ maps $D_{\omega}^{\mathbb{N}}$ into $D_{i}^{\mathbb{N}}$ ), and define $\Pi_{\omega}: D_{\omega}^{\mathbb{N}} \rightarrow D_{1}^{\mathbb{N}} \times \cdots \times D_{N}^{\mathbb{N}}$ by

$$
\Pi_{\omega}(\boldsymbol{i})=\left(\Pi_{\omega, 1}(\boldsymbol{i}), \ldots, \Pi_{\omega, N}(\boldsymbol{i})\right) .
$$

Lemma 4.3. Fix $q \in \mathbb{R}$. For $\omega \in U$, we have $\tilde{\mu}_{\omega}^{q} \circ \Pi_{\omega}^{-1}=\times_{i} \tilde{\mu}_{i}^{q}$. 
Proof. It clearly suffices to prove that if $n_{i}$ is a positive integer and $\boldsymbol{i}_{i} \in D_{i}^{n_{i}}$ for $i=1, \ldots, N$, then $\left(\tilde{\mu}_{\omega}^{q} \circ \Pi_{\omega}^{-1}\right)\left(\left[\boldsymbol{i}_{1}\right] \times \cdots \times\left[\boldsymbol{i}_{n}\right]\right)=\left(\times_{i} \tilde{\mu}_{i}^{q}\right)\left(\left[\boldsymbol{i}_{1}\right] \times \cdots \times\left[\boldsymbol{i}_{n}\right]\right)$. However, this equality follows immediately from the definition of $\Pi_{\omega}$.

Proposition 4.4. There is a set $W \subseteq \Omega$ such that

(1) $\mathbb{P}(W)=1$;

(2) for all $q \in \mathbb{R}$ and all $\omega \in W$, we have

$$
\lim _{r \backslash 0} \frac{\log \mu_{\omega}\left(Q_{\omega}(\boldsymbol{i}, r)\right)}{\log r}=-\beta^{\prime}(q)
$$

for $\tilde{\mu}_{\omega}^{q}$-almost all $\boldsymbol{i} \in D_{\omega}^{\mathbb{N}}$

(3) for all $q \in \mathbb{R}$ and all $\omega \in W$, we have

$$
\lim _{r \backslash 0} \frac{\log \mu_{\omega}^{q}\left(Q_{\omega}(\boldsymbol{i}, r)\right)}{\log r}=-q \beta^{\prime}(q)+\beta(q)
$$

for $\tilde{\mu}_{\omega}^{q}$-almost all $\boldsymbol{i} \in D_{\omega}^{\mathbb{N}}$.

Proof. Let $V$ be defined as in Lemma 3.2 and, for $\omega \in V$, let $r_{0}(\omega)$ be defined as in Lemma 3.2. Write $N_{\omega, i}(n)=\left|\left\{j \leq n \mid \omega_{j}=i\right\}\right|$ and put

$$
\begin{aligned}
& V_{1}=\left\{\omega \in \Omega \mid \lim _{n} \frac{N_{\omega, i}(n)}{n}=p_{i} \text { for all } i\right\}, \\
& V_{2}=\left\{\omega \in \Omega \mid \lim _{r>0} \frac{\log r}{k_{\omega, \ell}(r)}=-\log v_{\ell} \text { for all } \ell\right\} .
\end{aligned}
$$

(We note that $V_{1} \subseteq V_{2}$; however, for clarity we have decided to work with both $V_{1}$ and $V_{2}$.) Now let

$$
W=U \cap V \cap V_{1} \cap V_{2} .
$$

(The reader will notice that if $p_{i}>0$ for all $i$, then $V_{1} \subseteq U$.)

(1) It follows immediately from the ergodic theorem that $\mathbb{P}\left(V \cap V_{1} \cap V_{2}\right)=1$ (see Lemma 3.1 and Lemma 3.2), and since $\mathbb{P}(U)=1$, we conclude that $\mathbb{P}(W)=1$.

(2) Fix $q \in \mathbb{R}$ and $\omega=\left(\omega_{1}, \omega_{2}, \ldots\right) \in W$. We must now prove that

$$
\lim _{r \backslash 0} \log \frac{\mu_{\omega}\left(Q_{\omega}(\boldsymbol{i}, r)\right)}{\log r}=-\beta^{\prime}(q) \quad \text { for } \tilde{\mu}_{\omega}^{q} \text { almost all } \boldsymbol{i} \in D_{\omega}^{\mathbb{N}} .
$$

For $i=1, \ldots, N$ and $\ell=1, \ldots, d$, define $\Phi_{i, \ell}: D_{i}^{\mathbb{N}} \rightarrow \mathbb{R}$ by

$$
\Phi_{i, \ell}\left(\mathbf{i}_{1} \mathbf{i}_{2} \ldots\right)=p_{D_{i}, \mathbf{p}_{i}, \ell}\left(i_{1, \ell} \mid i_{1,1}, \ldots, i_{1, \ell-1}\right),
$$


where $\mathbf{i}_{1}=\left(i_{1,1}, \ldots, i_{1, d}\right)$. Let $S_{i}: D_{i}^{\mathbb{N}} \rightarrow D_{i}^{\mathbb{N}}$ denote the shift map on $D_{i}^{\mathbb{N}}$ and write

$$
M_{i}^{q}=\left\{\boldsymbol{i} \in D_{i}^{\mathbb{N}} \mid \lim _{n} \frac{1}{n} \sum_{j=1}^{n} \log \Phi_{i, \ell}\left(S_{i}^{j-1} \boldsymbol{i}\right)=\int \log \Phi_{i, \ell} \mathrm{d} \tilde{\mu}_{i}^{q} \text { for all } \ell\right\},
$$

and

$$
M_{\omega}^{q}=\Pi_{\omega}^{-1}\left(M_{1}^{q} \times \cdots \times M_{N}^{q}\right) .
$$

Claim 4.5. We have $\tilde{\mu}_{\omega}^{q}\left(M_{\omega}^{q}\right)=1$.

Proof of Claim 4.5. From the ergodic theorem, it immediately follows that $\tilde{\mu}_{i}^{q}\left(M_{i}\right)=1$ for all $i$, and since $\omega \in W \subseteq U$, Lemma 4.3 implies that

$$
\begin{aligned}
\tilde{\mu}_{\omega}^{q}\left(M_{\omega}^{q}\right) & =\tilde{\mu}_{\omega}^{q}\left(\Pi_{\omega}^{-1}\left(M_{1}^{q} \times \cdots \times M_{N}^{q}\right)\right)=\left(\times_{i} \tilde{\mu}_{i}\right)\left(M_{1}^{q} \times \cdots \times M_{N}^{q}\right) \\
& =\prod_{i} \tilde{\mu}_{i}^{q}\left(M_{i}^{q}\right)=1 .
\end{aligned}
$$

This completes the proof of Claim 4.5. $M_{\omega}^{q}$.

Claim 4.6. We have $\lim _{r \backslash 0}\left(\log \mu_{\omega}\left(Q_{\omega}(\boldsymbol{i}, r)\right) / \log r\right)=-\beta^{\prime}(q)$ for all $\boldsymbol{i} \in$

Proof of Claim 4.6. Fix $\boldsymbol{i} \in M_{\omega}^{q}$, and note that it follows from Lemma 3.3 that

$$
\begin{aligned}
\frac{\log \mu_{\omega}\left(Q_{\omega}(\boldsymbol{i}, r)\right)}{\log r} & =\frac{1}{\log r} \sum_{\ell=1}^{d} \sum_{j=1}^{k_{\omega, \ell}(r)} \log p_{\omega_{j}, \ell}\left(i_{j, \ell} \mid i_{j, 1}, \ldots, i_{j, \ell-1}\right) \\
& =\frac{1}{\log r} \sum_{i=1}^{N} \sum_{\ell=1}^{d} \sum_{\substack{j=1, \ldots, k_{\omega, \ell}(r) \\
\omega_{j}=i}} \log p_{\omega_{j}, \ell}\left(i_{j, \ell} \mid i_{j, 1}, \ldots, i_{j, \ell-1}\right) \\
& =\frac{1}{\log r} \sum_{i=1}^{N} \sum_{\ell=1}^{d} \sum_{\substack{j=1, \ldots, k_{\omega, \ell}(r) \\
\omega_{j}=i}} \log p_{\omega_{j}, \ell}\left(i_{j, \ell} \mid i_{j, 1}, \ldots, i_{j, \ell-1}\right) .
\end{aligned}
$$

Recall that, for each positive integer $n$, we write $N_{\omega, i}(n)=\left|\left\{j \leq n \mid \omega_{j}=i\right\}\right|$. Since $\Phi_{i, \ell}\left(\mathbf{j}_{1} \mathbf{j}_{2} \ldots\right)$ only depends on $\mathbf{j}_{1}$, we conclude that if $\omega_{j}=i$, then

$$
p_{i, \ell}\left(i_{j, \ell} \mid i_{j, 1}, \ldots, i_{j, \ell-1}\right)=\Phi_{i, \ell}\left(S_{i}^{N_{\omega, i}(j)-1} \Pi_{\omega, i}(\boldsymbol{i})\right) .
$$


Hence,

$$
\begin{aligned}
\frac{\log \mu_{\omega}\left(Q_{\omega}(\boldsymbol{i}, r)\right)}{\log r}= & \frac{1}{\log r} \sum_{i=1}^{N} \sum_{\ell=1}^{d} \sum_{\substack{j=1, \ldots, k_{\omega, \ell}(r) \\
\omega_{j}=i}} \log \Phi_{i, \ell}\left(S_{i}^{N_{\omega, i}(j)-1} \Pi_{\omega, i}(\boldsymbol{i})\right) \\
= & \frac{1}{\log r} \sum_{i=1}^{N} \sum_{\ell=1}^{d} \sum_{j=1}^{N_{\omega, i}\left(k_{\omega, \ell}(r)\right)} \log \Phi_{i, \ell}\left(S_{i}^{j-1} \Pi_{\omega, i}(\boldsymbol{i})\right) \\
= & \sum_{i=1}^{N} \sum_{\ell=1}^{d} \frac{k_{\omega, \ell}(r)}{\log r} \frac{N_{\omega, i}\left(k_{\omega, \ell}(r)\right)}{k_{\omega, \ell}(r)} \\
& \times \frac{1}{N_{\omega, i}\left(k_{\omega, \ell}(r)\right)} \sum_{j=1}^{N_{\omega, i}\left(k_{\omega, \ell}(r)\right)} \log \Phi_{i, \ell}\left(S_{i}^{j-1} \Pi_{\omega, i}(\boldsymbol{i})\right) .
\end{aligned}
$$

Next, since $\boldsymbol{i} \in M_{\omega}^{q}$, we deduce that $\Pi_{\omega, i}(\boldsymbol{i}) \in M_{i}^{q}$, whence

$$
\frac{1}{n} \sum_{j=1}^{n} \log \Phi_{i, \ell}\left(S_{i}^{j-1} \Pi_{\omega, i}(\boldsymbol{i})\right) \rightarrow \int \log \Phi_{i, \ell} \mathrm{d} \tilde{\mu}_{i}^{q}
$$

as $n \rightarrow \infty$. Also, since $\omega \in W \subseteq V_{1} \cap V_{2}$, we conclude that $N_{\omega, i}(n) / n \rightarrow p_{i}$ as $n \rightarrow \infty$ and $\log r / k_{\omega, \ell}(r) \rightarrow-\log \nu_{\ell}$ as $r \succ 0$. Combining this with (4.7) and (4.8) yields

$$
\frac{\log \mu_{\omega}\left(Q_{\omega}(\boldsymbol{i}, r)\right)}{\log r} \rightarrow-\sum_{i=1}^{N} \sum_{\ell=1}^{d} \frac{1}{\log \nu_{\ell}} p_{i} \int \log \Phi_{i, \ell} \mathrm{d} \tilde{\mu}_{i}^{q}
$$

as $r \backslash 0$. Next, we compute the integral $\int \log \Phi_{i, \ell} \mathrm{d} \tilde{\mu}_{i}^{q}$. Indeed, we clearly have

$$
\begin{aligned}
\int \log \Phi_{i, \ell} \mathrm{d} \tilde{\mu}_{i}^{q} & =\sum_{\mathbf{i}=\left(i_{1}, \ldots, i_{d}\right) \in D_{i}} P_{i, \mathbf{i}}(q) \log p_{i, \ell}\left(i_{\ell} \mid i_{1}, \ldots, i_{\ell-1}\right) \\
& =\sum_{\left(i_{1}, \ldots, i_{\ell}\right) \in \pi_{\ell}\left(D_{i}\right)}\left(\sum_{\substack{\mathbf{j}=\left(j_{1}, \ldots, j_{d}\right) \in D_{i} \\
j_{1}=i_{1}, \ldots, j_{\ell}=i_{\ell}}} P_{i, \mathbf{j}}(q)\right) \log p_{i, \ell}\left(i_{\ell} \mid i_{1}, \ldots, i_{\ell-1}\right) \\
& =\sum_{\left(i_{1}, \ldots, i_{\ell}\right) \in \pi_{\ell}\left(D_{i}\right)} Q_{i, \ell}\left(q ; i_{1}, \ldots, i_{\ell}\right) \log p_{D_{i}, \mathbf{p}_{i}, \ell}\left(i_{\ell} \mid i_{1}, \ldots, i_{\ell-1}\right) .
\end{aligned}
$$

It follows immediately from (4.9), (4.10) and Lemma 4.1 (1) that 


$$
\begin{aligned}
\frac{\log \mu_{\omega}\left(Q_{\omega}(\boldsymbol{i}, r)\right)}{\log r} & \rightarrow-\sum_{i=1}^{N} \sum_{\ell=1}^{d} \frac{1}{\log } v_{\ell} p_{i} \sum_{\left(i_{1}, \ldots, i_{\ell}\right) \in \pi_{\ell}\left(D_{i}\right)} Q_{i, \ell}\left(q ; i_{1}, \ldots, i_{\ell}\right) \\
\times \log p_{D_{i}, \mathbf{p}_{i}, \ell}\left(i_{\ell} \mid i_{1}, \ldots, i_{\ell-1}\right) & \\
& =-\beta^{\prime}(q)
\end{aligned}
$$

for all $\boldsymbol{i} \in M_{\omega}^{q}$ as $r \searrow 0$. This completes the proof of Claim 4.6.

The desired result now follows immediately from Claim 4.5 and Claim 4.6.

(3) The proof of (3) is similar to the proof of (2) using Lemma 4.1 (2) instead of Lemma 4.1 (1), and is therefore omitted.

Finally, we turn towards the proof of (1.33). In order to prove (1.33) using Proposition 4.4, we need the following version of a standard result due to Cutler $[\mathrm{C}]$ and Young $[\mathrm{Y}]$. However, first recall that if $\mu$ is a probability measure on $\mathbb{R}^{d}$, then the Hausdorff dimension $\operatorname{dim}_{H}(\mu)$ of $\mu$ is defined by

$$
\operatorname{dim}_{H}(\mu)=\inf _{\mu(E)=1} \operatorname{dim}_{H}(E) .
$$

We can now state the version of the result due to Cutler $[\mathrm{C}]$ and Young $[\mathrm{Y}]$ that we need.

Proposition 4.7. For each $\omega=\left(\omega_{1}, \omega_{2}, \ldots\right), \ell \in\{1, \ldots, d\}$ and $x \in \mathbb{R}$, let

$$
x=\sum_{i=1}^{\infty} \frac{\varepsilon_{\omega, i, \ell}(x)}{n_{\omega_{1}, \ell} n_{\omega_{2}, \ell} \cdots n_{\omega_{i}, \ell}},
$$

with $\varepsilon_{\omega, i, \ell}(x) \in\left\{0,1, \ldots, n_{\omega_{i}, \ell}-1\right\}$, denote the unique non-terminating

$$
\left(\frac{1}{n_{\omega_{1}, \ell}}, \frac{1}{n_{\omega_{1}, \ell} n_{\omega_{2}, \ell}}, \frac{1}{n_{\omega_{1}, \ell} n_{\omega_{2}, \ell} n_{\omega_{3}, \ell}}, \ldots\right) \text {-expansion of } x .
$$

For $x=\left(x_{1}, \ldots, x_{d}\right) \in \mathbb{R}^{d}$ write

$$
\boldsymbol{\varepsilon}_{\omega, i}(x)=\left(\varepsilon_{\omega, i, 1}\left(x_{1}\right), \ldots, \varepsilon_{\omega, i, d}\left(x_{d}\right)\right) \in \Sigma_{\omega_{i}}
$$

(recall that $\Sigma_{\omega_{i}}$ is defined in Section 1.3.1) and put

$$
\boldsymbol{\varepsilon}_{\omega}(x)=\left(\boldsymbol{\varepsilon}_{\omega, 1}(x), \boldsymbol{\varepsilon}_{\omega, 2}(x), \ldots\right) \in \Sigma_{\omega}^{\mathbb{N}}
$$

(recall that $\Sigma_{\omega}^{\mathbb{N}}$ is defined in Section 1.3.1). Let $\mu$ be a probability measure on $\mathbb{R}^{d}$ and $\alpha \geq 0$. Assume that

$$
\lim _{r>0} \frac{\log \mu\left(Q_{\omega}\left(\varepsilon_{\omega}(x), r\right)\right)}{\log r}=\alpha
$$

for $\mu$-almost all $x$. Then

$$
\operatorname{dim}_{H}(\mu)=\alpha
$$


Proof. This result is proved in, e.g., [C; H, Proposition 1; M1, Theorem 6.9 and Theorem 6.11] or [Y] using balls in place of approximate cubes. However, if we write $n_{\max }=\max _{i, \ell} n_{i, \ell}$ and $Q_{\omega}(\boldsymbol{i}, r)=\mathrm{X}_{\ell=1}^{d} I_{\omega, \ell}(\boldsymbol{i}, r)$, then (see (1.31)) $1 / n_{\max } \leq \operatorname{diam} I_{\omega, \ell_{1}}(\boldsymbol{i}, r) / \operatorname{diam} I_{\omega, \ell_{2}}(\boldsymbol{i}, r) \leq n_{\max }$ for all $\ell_{1}, \ell_{2}=1, \ldots, d$, i.e., the ratio between the diameters of the intervals $I_{\omega, \ell}(\boldsymbol{i}, r)$ is bounded below and above, and we can thus in the definitions of the Hausdorff measure in [C, H, M1, $\mathrm{Y}]$ restrict our attention to covers by approximate cubes.

We can now prove (1.33). Recall that the numbers $\alpha_{\min }$ and $\alpha_{\max }$ are defined in (1.29).

Theorem 4.8. For $\mathbb{P}$-almost all $\omega \in \Omega$ we have

$$
\beta^{*}(\alpha) \leq f_{\mathrm{H}, \mu_{\omega}}^{\mathrm{sym}}(\alpha)
$$

for all $\alpha \in\left(\alpha_{\min }, \alpha_{\max }\right)$.

Proof. For $\omega \in \Omega$ and $\alpha \in \mathbb{R}$, write

$$
\Delta_{\omega}(\alpha)=\left\{\boldsymbol{i} \in D_{\omega}^{\mathbb{N}} \mid \lim _{r>0} \frac{\log \mu_{\omega}\left(Q_{\omega}(\boldsymbol{i}, r)\right)}{\log r}=\alpha\right\} .
$$

Let $W$ be as in Proposition 4.4. Next, fix $\omega \in W$ and $\alpha \in\left(\alpha_{\min }, \alpha_{\max }\right)$. Since $\alpha \in\left(\alpha_{\min }, \alpha_{\max }\right)$, we conclude from Lemma 4.2 that there is $q \in \mathbb{R}$ such that $-\beta^{\prime}(q)=\alpha$. It therefore follows from Proposition 4.4 and Proposition 4.7 that we have

$$
\begin{array}{rlrl}
f_{\mathrm{H}, \mu_{\omega}}^{\mathrm{sym}}(\alpha) & =f_{\mathrm{H}, \mu_{\omega}}^{\mathrm{sym}}\left(-\beta^{\prime}(q)\right) & & \\
& =\operatorname{dim}_{\mathrm{H}}\left(\pi_{\omega} \Delta_{\omega}\left(-\beta^{\prime}(q)\right)\right) & & \\
& \geq \operatorname{dim}_{\mathrm{H}}\left(\tilde{\mu}_{\omega}^{q} \circ \pi_{\omega}^{-1}\right) & & {[\text { by Proposition 4.4(2)] }} \\
& =\operatorname{dim}_{\mathrm{H}}\left(\mu_{\omega}^{q}\right) & & {\left[\text { since } \mu_{\omega}^{q}=\tilde{\mu}_{\omega}^{q} \circ \pi_{\omega}^{-1}\right]} \\
& =-q \beta^{\prime}(q)+\beta(q) & & {[\text { by Proposition 4.4 (3)] }} \\
& =\beta^{*}\left(-\beta^{\prime}(q)\right)=\beta^{*}(\alpha) . &
\end{array}
$$

This completes the proof.

5. Proof of the Upper Bound (1.34) In Theorem 1.2

The purpose of this section is to prove the upper bound (1.34) in Theorem 1.2.

Fix $q \in \mathbb{R}$. For $\omega \in \Omega$ and $\boldsymbol{i} \in D_{\omega}^{\mathbb{N}}$, define the density $D_{\omega}^{q}(\boldsymbol{i}, r)$ by

$$
D_{\omega}^{q}(\boldsymbol{i}, r)=\frac{\mu_{\omega}^{q}\left(Q_{\omega}(\boldsymbol{i}, r)\right)}{\mu_{\omega}\left(Q_{\omega}(\boldsymbol{i}, r)\right)^{q} r^{\beta(q)}} .
$$


The proof of (1.34) is divided into two parts:

(1) Firstly, we show that for $\mathbb{P}$-almost all $\omega \in \Omega$, the limiting density given by $\lim \sup _{r \backslash 0} D_{\omega}^{q}(\boldsymbol{i}, r)^{1 /-\log r}$ is greater than or equal to 1 for all $q \in \mathbb{R}$ and all $\boldsymbol{i} \in D_{\omega}^{\mathbb{N}}$; i.e., we show that for $\mathbb{P}$-almost all $\omega \in \Omega$, we have

$$
\underset{r \succ 0}{\limsup } D_{\omega}^{q}(\boldsymbol{i}, r)^{1 /(-\log r)} \geq 1
$$

for all $q \in \mathbb{R}$ and all $\boldsymbol{i} \in D_{\omega}^{\mathbb{N}}$. The proof of this inequality is fairly technical and is divided into several propositions-namely, Propositions 5.1-5.5.

(2) Secondly, we apply inequality (5.2) and a suitable version of the so-called mass distribution principle to prove (1.34). This is done in Proposition 5.6 and Theorems 5.7-5.8.

We now turn towards the proof of inequality (5.2). We begin by introducing some notation. Write

$$
\theta_{\ell}=\frac{\log v_{\ell}}{\log v_{\ell+1}}=\frac{\sum_{i} p_{i} \log n_{i, \ell}}{\sum_{i} p_{i} \log n_{i, \ell+1}} \text { for } \ell=1, \ldots, d-1 .
$$

Next, recall that we write

$$
N_{\omega, i}(n)=\left|\left\{j \leq n \mid \omega_{j}=i\right\}\right|,
$$

and for $\omega=\left(\omega_{1}, \omega_{2}, \ldots\right) \in \Omega$ and $\boldsymbol{i}=\mathbf{i}_{1} \mathbf{i}_{2} \ldots \in D_{\omega}^{\mathbb{N}}$ with $\mathbf{i}_{j}=\left(i_{j, 1}, \ldots, i_{j, d}\right)$, let

$$
h_{\omega, i, \ell}(q ; \boldsymbol{i} ; n)=\left(\prod_{\substack{j \leq n \\ \omega_{j}=i}} v_{\ell}^{b_{i, i}, \ldots, i_{j, \ell}(q)}\right)^{\left(1 / N_{\omega, i}(n)\right)\left(p_{i} / \log v_{\ell}\right)} .
$$

Note that if we define $h_{\min }(q)$ and $h_{\max }(q)$ by

$$
h_{\min }(q)=\min _{i} \min _{\ell} \min _{\left(i_{1}, \ldots, i_{d}\right) \in D_{i}} v_{\ell}^{b_{i, i_{1} \ldots i_{\ell}}(q) p_{i} / \log v_{\ell}}
$$

and

$$
h_{\max }(q)=\max _{i} \max _{\ell} \max _{\left(i_{1}, \ldots, i_{d}\right) \in D_{i}} v_{\ell}^{b_{i, i_{1} \ldots i_{\ell}}(q) p_{i} / \log v_{\ell}},
$$

then

$$
0<h_{\min }(q) \leq h_{\omega, i, \ell}(q ; i ; n) \leq h_{\max }(q)<\infty .
$$


Proposition 5.1. Fix $q \in \mathbb{R}$. Let $\omega \in V$ (where $V$ is defined in Lemma 3.2) and $\boldsymbol{i} \in D_{\omega}^{\mathbb{N}}$. Then

$$
\begin{aligned}
& D_{\omega}^{q}(\boldsymbol{i}, r)=e^{-\left(\beta(q)-\sum_{i=1}^{N} \log v_{1}\left(N_{\omega, i}\left(k_{\omega, 1}(r)\right) /(-\log r)\right) b_{i}(q)\right) \log r} \\
& \times\left(\prod_{i=1}^{N} \prod_{\ell=1}^{d-1}\left(\frac{h_{\omega, i, \ell}\left(q ; \boldsymbol{i} ; k_{\omega, \ell}(r)\right)}{h_{\omega, i, \ell}\left(q ; \boldsymbol{i} ; k_{\omega, \ell+1}(r)\right)}\right)^{N_{\omega, i}\left(k_{\omega, \ell}(r)\right) \log v_{\ell} / p_{i}}\right) \\
& \times\left(\prod_{i=1}^{N} \prod_{\ell=1}^{d-1} h_{\omega, i, \ell}\left(q ; \boldsymbol{i} ; k_{\omega, \ell+1}(r)\right)^{\left(1-\frac{1}{\theta_{\ell}} \frac{N_{\omega, i}\left(k_{\omega, \ell+1}(r)\right)}{N_{\omega, i^{(}}\left(k_{\omega, \ell^{(r))}}\right)}\right)_{\omega, i}\left(k_{\omega, \ell}(r)\right) \log v_{\ell} / p_{i}}\right)
\end{aligned}
$$

for all $0<r<r_{0}(\omega)$ (where $r_{0}(\omega)$ is defined in Lemma 3.2).

Proof. Write $\omega=\left(\omega_{1}, \omega_{2}, \ldots\right) \in \Omega$, and

$$
\boldsymbol{i}=\mathbf{i}_{1} \mathbf{i}_{2} \ldots \in D_{\omega}^{\mathbb{N}} \quad \text { with } \quad \mathbf{i}_{j}=\left(i_{j, 1}, \ldots, i_{j, d}\right) \in D_{\omega_{j}} .
$$

Then it follows from Lemma 3.3 and Lemma 3.4 that

$$
\begin{aligned}
D_{\omega}^{q}(\boldsymbol{i}, r) & =\frac{\mu_{\omega}^{q}\left(Q_{\omega}(\boldsymbol{i}, r)\right)}{\mu_{\omega}\left(Q_{\omega}(\boldsymbol{i}, r)\right)^{q} r^{\beta(q)}} \\
& =\frac{1}{r^{\beta(q)}} \frac{\prod_{\ell=1}^{d} \prod_{j=1}^{k_{\omega, \ell}(r)} P_{\omega_{j, \ell}}\left(q ; i_{j, \ell} \mid i_{j, 1}, \ldots, i_{j, \ell-1}\right)}{\prod_{\ell=1}^{k_{\omega, \ell}(r)} \prod_{j=1}^{k_{j}} p_{\omega_{j}, \ell}\left(i_{j, \ell} \mid i_{j, 1}, \ldots, i_{j, \ell-1}\right)^{q}} \\
& =\frac{1}{r^{\beta(q)}} \prod_{\ell=1}^{d} \prod_{j=1}^{k_{\omega, \ell}(r)} \frac{P_{\omega_{j}, \ell}\left(q ; i_{j, \ell} \mid i_{j, 1}, \ldots, i_{j, \ell-1}\right)}{p_{\omega_{j}, \ell}\left(i_{j, \ell} \mid i_{j, 1}, \ldots, i_{j, \ell-1}\right)^{q}} .
\end{aligned}
$$

Momentarily writing

$$
\Lambda_{j, \ell}=\frac{P_{\omega_{j}, \ell}\left(q ; i_{j, \ell} \mid i_{j, 1}, \ldots, i_{j, \ell-1}\right)}{p_{\omega_{j}, \ell}\left(i_{j, \ell} \mid i_{j, 1}, \ldots, i_{j, \ell-1}\right)^{q}}
$$

and noticing that $k_{\omega, 1}(r) \geq k_{\omega, 2}(r) \geq \cdots \geq k_{\omega, d}(r)$ because $\omega \in V$ and $0<r<r_{0}(\omega)$ (see Lemma 3.2), we see that 


$$
\begin{aligned}
\prod_{\ell=1}^{d} \prod_{j=1}^{k_{\omega, \ell}(r)} & \frac{P_{\omega_{j}, \ell}\left(q ; i_{j, \ell} \mid i_{j, 1}, \ldots, i_{j, \ell-1}\right)}{p_{\omega_{j}, \ell}\left(i_{j, \ell} \mid i_{j, 1}, \ldots, i_{j, \ell-1}\right)^{q}}=\prod_{\ell=1}^{d} \prod_{j=1}^{k_{\omega, \ell}(r)} \Lambda_{j, \ell} \\
= & \Lambda_{1,1} \cdots \Lambda_{k_{\omega, d}(r), 1} \Lambda_{k_{\omega, d}(r)+1,1} \cdots \Lambda_{k_{\omega, d-1}(r), 1} \cdots \\
& \quad \times \cdots \Lambda_{k_{\omega, 3}(r)+1,1} \cdots \Lambda_{k_{\omega, 2}(r), 1} \Lambda_{k_{\omega, 2}(r)+1,1} \cdots \Lambda_{k_{\omega, 1}(r), 1} \\
& \times \Lambda_{1,2} \cdots \Lambda_{k_{\omega, d}(r), 2} \Lambda_{k_{\omega, d}(r)+1,2} \cdots \Lambda_{k_{\omega, d-1}(r), 2} \cdots \\
& \times \cdots \Lambda_{k_{\omega, 3}(r)+1,2} \cdots \Lambda_{k_{\omega, 2}(r), 2} \\
& \quad \vdots \\
& \times \Lambda_{1, d} \cdots \Lambda_{k_{\omega, d}}(r), d \\
= & \left(\prod_{s=1}^{d} \Lambda_{1, s}\right) \cdots\left(\prod_{s=1}^{d} \Lambda_{k_{\omega, d}(r), s}\right) \\
& \times\left(\prod_{s=1}^{d-1} \Lambda_{k_{\omega, d}(r)+1, s}\right) \cdots\left(\prod_{s=1}^{d-1} \Lambda_{k_{\omega, d-1}(r), s}\right) \\
& \quad \vdots \\
& \times\left(\prod_{s=1}^{2} \Lambda_{k_{\omega, 3}(r)+1, s}\right) \cdots\left(\prod_{s=1}^{2} \Lambda_{k_{\omega, 2}(r), s}\right) \\
& \times\left(\prod_{s=1}^{1} \Lambda_{k_{\omega, 2}(r)+1, s} \cdots\left(\prod_{s=1}^{1} \Lambda_{k_{\omega, 1}(r), s}\right) .\right.
\end{aligned}
$$

Next, we consider the $(d-\ell+1)$ 'th "row" in (5.6). Indeed, note that this "row" can be rewritten as

$$
\begin{aligned}
& \left(\prod_{s=1}^{\ell} \Lambda_{k_{\omega, \ell+1}(r)+1, s}\right) \cdots\left(\prod_{s=1}^{\ell} \Lambda_{k_{\omega, \ell}(r), s}\right)=\prod_{j=k_{\omega, \ell+1}(r)+1}^{k_{\omega, \ell}(r)} \prod_{s=1}^{\ell} \Lambda_{j, s} \\
& =\prod_{j=k_{\omega, \ell+1}(r)+1}^{k_{\omega, \ell}(r)} \prod_{s=1}^{\ell} \frac{P_{\omega_{j}, s}\left(q ; i_{j, s} \mid i_{j, 1}, \ldots, i_{j, s-1}\right)}{p_{\omega_{j}, s}\left(i_{j, s} \mid i_{j, 1}, \ldots, i_{j, s-1}\right)^{q}} \\
& =\prod_{j=k_{\omega, \ell+1}(r)+1}^{k_{\omega, \ell}(r)} \frac{\prod_{s=1}^{\ell} P_{\omega_{j, s}}\left(q ; i_{j, s} \mid i_{j, 1}, \ldots, i_{j, s-1}\right)}{\prod_{s=1}^{\ell} p_{\omega_{j}, s}\left(i_{j, s} \mid i_{j, 1}, \ldots, i_{j, s-1}\right)^{q}} \\
& =\prod_{j=k_{\omega, \ell+1}(r)+1}^{k_{\omega, \ell}(r)} \frac{Q_{\omega_{j}, \ell}\left(q ; i_{j, 1}, \ldots, i_{j, \ell}\right)}{q_{\omega_{j}, \ell}\left(i_{j, 1}, \ldots, i_{j, \ell}\right)^{q}} .
\end{aligned}
$$


For brevity, we introduce the following notation. Namely, we will write

$$
\begin{array}{ll}
u_{j, \ell}=v_{\ell} b_{\omega_{j, i}, \ldots} \ldots i_{j, \ell}(q) & \text { for } \ell=1, \ldots, d, \\
v_{j, \ell}=v_{\ell+1}^{b_{\omega_{j}, i_{j, 1} \ldots i_{j, \ell}}(q)} & \text { for } \ell=0, \ldots, d-1,
\end{array}
$$

and

$$
\begin{array}{ll}
U_{n, \ell}=u_{1, \ell} \cdots u_{n, \ell} & \text { for } \ell=1, \ldots, d, \\
V_{n, \ell}=v_{1, \ell} \cdots v_{n, \ell} & \text { for } \ell=0, \ldots, d-1 .
\end{array}
$$

Using Lemma 3.5 we have

$$
\begin{aligned}
& \frac{Q_{\omega_{j}, \ell}\left(q ; i_{j, 1}, \ldots, i_{j, \ell}\right)}{q_{\omega_{j, \ell}}\left(i_{j, 1}, \ldots, i_{j, \ell}\right)^{q}} \\
& =v_{\ell}^{b_{\omega_{j}, i_{j, 1} \ldots i_{j, \ell}}(q)-b_{\omega_{j}, i_{j, 1} \ldots i_{j, \ell-1}}(q)} \cdots v_{2}^{b_{\omega_{j, i}, i_{j, 1} i_{j, 2}}(q)-b_{\omega_{j}, i_{j, 1}}(q)} v_{1}^{b_{\omega_{j}, i_{j, 1}}(q)-b_{\omega_{j}}(q)} \\
& =\frac{v_{\ell}^{b_{\omega_{j}, i_{j, 1} \ldots i_{j, \ell}}(q)}}{v_{\ell}^{b_{\omega_{j}, i_{j, 1} \ldots i_{j, \ell-1}}(q)}} \cdots \frac{v_{2}^{b_{\omega_{j}, i_{j, 1} i_{j, 2}}(q)}}{v_{2}^{b_{\omega_{j}, i_{j, 1}}(q)}} \frac{v_{1}^{b_{\omega_{j}, i_{j, 1}}(q)}}{v_{1}^{b_{\omega_{j}}(q)}} \\
& =\frac{u_{j, \ell}}{v_{j, \ell-1}} \cdots \frac{u_{j, 2}}{v_{j, 1}} \frac{u_{j, 1}}{v_{j, 0}} \text {. }
\end{aligned}
$$

Combining (5.7) and (5.8) now shows that the $(d-\ell+1)$ 'th "row" in (5.6) can be rewritten as

$$
\begin{aligned}
& \left(\prod_{s=1}^{\ell} \Lambda_{k_{\omega, \ell+1}(r)+1, s}\right) \ldots\left(\prod_{s=1}^{\ell} \Lambda_{k_{\omega, \ell}(r), s}\right) \\
& =\prod_{j=k_{\omega, \ell+1}(r)+1}^{k_{\omega, \ell}(r)}\left(\frac{u_{j, \ell}}{v_{j, \ell-1}} \cdots \frac{u_{j, 2}}{v_{j, 1}} \frac{u_{j, 1}}{v_{j, 0}}\right) \\
& k_{\omega, \ell}(r) \quad k_{\omega, \ell}(r) \quad k_{\omega, \ell}(r) \\
& \prod u_{j, \ell} \prod u_{j, 2} \prod u_{j, 1} \\
& =\frac{j=k_{\omega, \ell+1}(r)+1}{k_{\omega, \ell}(r)} \cdots \frac{j=k_{\omega, \ell+1}(r)+1}{k_{\omega, \ell}(r)} \frac{j=k_{\omega, \ell+1}(r)+1}{k_{\omega, \ell}(r)} \\
& \prod_{j=k_{\omega, \ell+1}(r)+1} v_{j, \ell-1} \prod_{j=k_{\omega, \ell+1}(r)+1} v_{j, 1} \prod_{j=k_{\omega, \ell+1}(r)+1} v_{j, 0} \\
& =\frac{\left(\frac{U_{k_{\omega, \ell}(r), \ell}}{U_{k_{\omega, \ell+1}}(r), \ell}\right)}{\left(\frac{V_{k_{\omega, \ell}}(r), \ell-1}{V_{k_{\omega, \ell+1}(r), \ell-1}}\right)} \cdots \frac{\left(\frac{U_{k_{\omega, \ell}(r), 2}}{U_{k_{\omega, \ell+1}}(r), 2}\right)}{\left(\frac{V_{k_{\omega, \ell}}(r), 1}{V_{k_{\omega, \ell+1}}(r), 1}\right)} \frac{\left(\frac{U_{k_{\omega, \ell}}(r), 1}{U_{k_{\omega, \ell+1}}(r), 1}\right)}{\left(\frac{V_{k_{\omega, \ell}}(r), 0}{V_{k_{\omega, \ell+1}}(r), 0}\right)} .
\end{aligned}
$$


Finally, substituting (5.9) into (5.6) gives

$$
\begin{aligned}
& \prod_{\ell=1}^{d} \prod_{j=1}^{k_{\omega, \ell}(r)} \frac{P_{\omega_{j}, \ell}\left(q ; i_{j, \ell} \mid i_{j, 1}, \ldots, i_{j, \ell-1}\right)}{p_{\omega_{j}, \ell}\left(i_{j, \ell} \mid i_{j, 1}, \ldots, i_{j, \ell-1}\right)^{q}} \\
& =\frac{U_{k_{\omega, d}(r), d}}{V_{k_{\omega, d}(r), d-1}} \frac{U_{k_{\omega, d}(r), d-1}}{V_{k_{\omega, d}(r), d-2}} \cdots \frac{U_{k_{\omega, d}}(r), 2}{V_{k_{\omega, d}}(r), 1} \frac{U_{k_{\omega, d}}(r), 1}{V_{k_{\omega, d}}(r), 0} \\
& \times \frac{\left(\frac{U_{k_{\omega, d-1}}(r), d-1}{U_{k_{\omega, d}}(r), d-1}\right)}{\left(\frac{V_{k_{\omega, d-1}}(r), d-2}{V_{k_{\omega, d}}(r), d-2}\right)} \cdots \frac{\left(\frac{U_{k_{\omega, d-1}}(r), 2}{U_{k_{\omega, d}}(r), 2}\right)}{\left(\frac{V_{k_{\omega, d-1}}(r), 1}{V_{k_{\omega, d}}(r), 1}\right)} \frac{\left(\frac{U_{k_{\omega, d-1}}(r), 1}{U_{k_{\omega, d}}(r), 1}\right)}{\left(\frac{V_{k_{\omega, d-1}}(r), 0}{V_{k_{\omega, d}}(r), 0}\right)} \\
& \times \frac{\left(\frac{U_{k_{\omega, 2}(r), 2}}{U_{k_{\omega, 3}}(r), 2}\right)}{\left(\frac{V_{k_{\omega, 2}}(r), 1}{V_{k_{\omega, 3}}(r), 1}\right)} \frac{\left(\frac{U_{k_{\omega, 2}(r), 1}}{U_{k_{\omega, 3}}(r), 1}\right)}{\left(\frac{V_{k_{\omega, 2}}(r), 0}{V_{k_{\omega, 3}}(r), 0}\right)} \\
& \times \frac{\left(\frac{U_{k_{\omega, 1}(r), 1}}{U_{k_{\omega, 2}}(r), 1}\right)}{\left(\frac{V_{k_{\omega, 1}}(r), 0}{V_{k_{\omega, 2}(r), 0}}\right)} .
\end{aligned}
$$

After cancellation this simplifies to

$\prod_{\ell=1}^{d} \prod_{j=1}^{k_{\omega, \ell}(r)} \frac{P_{\omega_{j}, \ell}\left(q ; i_{j, \ell} \mid i_{j, 1}, \ldots, i_{j, \ell-1}\right)}{p_{\omega_{j}, \ell}\left(i_{j, \ell} \mid i_{j, 1}, \ldots, i_{j, \ell-1}\right)^{q}}=U_{k_{\omega, d}(r), d}\left(\prod_{\ell=1}^{d-1} \frac{U_{k_{\omega, \ell}(r), \ell}}{V_{k_{\omega, \ell+1}(r), \ell}}\right) \frac{1}{V_{k_{\omega, 1}(r), 0}}$,

whence, using (5.5),

$$
\begin{aligned}
D_{\omega}^{q}(\boldsymbol{i}, r) & =\frac{1}{r^{\beta(q)}} \prod_{\ell=1}^{d} \prod_{j=1}^{k_{\omega, \ell}(r)} \frac{P_{\omega_{j}, \ell}\left(q ; i_{j, \ell} \mid i_{j, 1}, \ldots, i_{j, \ell-1}\right)}{p_{\omega_{j}, \ell}\left(i_{j, \ell} \mid i_{j, 1}, \ldots, i_{j, \ell-1}\right)^{q}} \\
& =\frac{1}{r^{\beta(q)}} U_{k_{\omega, d}(r), d}\left(\prod_{\ell=1}^{d-1} \frac{U_{k_{\omega, \ell}(r), \ell}}{V_{k_{\omega, \ell+1}(r), \ell}}\right) \frac{1}{V_{k_{\omega, 1}(r), 0}} \\
& =\frac{1}{r^{\beta(q)}} \frac{1}{V_{k_{\omega, 1}(r), 0}}\left(\prod_{\ell=1}^{d-1} \frac{U_{k_{\omega, \ell}(r), \ell}}{V_{k_{\omega, \ell+1}(r), \ell}}\right) .
\end{aligned}
$$


The right hand side of (5.10) consists of two terms — namely,

$$
\frac{1}{r^{\beta(q)}} \frac{1}{V_{k_{\omega, 1}(r), 0}} \text { and } \quad \prod_{\ell=1}^{d-1} \frac{U_{k_{\omega, \ell}(r), \ell}}{V_{k_{\omega, \ell+1}}(r), \ell} \text {. }
$$

We will now analyse those terms.

We first analyse the term $\left(1 / r^{\beta(q)}\right)\left(1 / V_{k_{\omega, 1}(r), 0}\right)$. Indeed, we clearly have

$$
\begin{aligned}
\frac{1}{r^{\beta(q)}} \frac{1}{V_{k_{\omega, 1}(r), 0}} & =\frac{1}{r^{\beta(q)}} \frac{1}{v_{1,0} \cdots v_{k_{\omega, 1}(r), 0}} \\
& =\frac{1}{r^{\beta(q)}} \frac{1}{v_{1}^{b_{\omega_{1}}(q)} \cdots v_{1}^{b_{\omega_{\omega, 1}}(r)}(q)} \\
& =e^{-\left(\beta(q)-\sum_{i=1}^{N} \log v_{1}\left(N_{\omega, i}\left(k_{\omega, 1}(r)\right) /(-\log r)\right) b_{i}(q)\right) \log r} .
\end{aligned}
$$

Next, we analyse the product $\prod_{\ell=1}^{d-1} U_{k_{\omega, \ell}(r), \ell} / V_{k_{\omega, \ell+1}(r), \ell}$. Using the fact that $v_{n, \ell}=u_{n, \ell}^{1 / \theta_{\ell}}$ for all positive integers $n$, we have

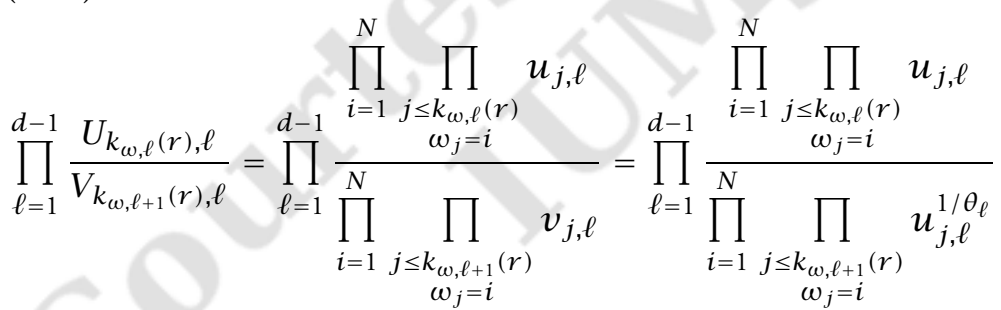

$$
\begin{aligned}
& =\prod_{\ell=1}^{d-1} \prod_{i=1}^{N} \frac{h_{\omega, i, \ell}\left(q ; \boldsymbol{i} ; k_{\omega, \ell}(r)\right)^{N_{\omega, i}\left(k_{\omega, \ell}(r)\right) \log v_{\ell} / p_{i}}}{h_{\omega, i, \ell}\left(q ; \boldsymbol{i} ; k_{\omega, \ell+1}(r)\right)^{N_{\omega, i}\left(\left(k_{\omega, \ell+1}(r)\right) / \theta_{\ell}\right)\left(\log v_{\ell} / p_{i}\right)}} \\
& =\left(\prod_{i=1}^{N} \prod_{\ell=1}^{d-1}\left(\frac{h_{\omega, i, \ell}\left(q ; \boldsymbol{i} ; k_{\omega, \ell}(r)\right)}{h_{\omega, i, \ell}\left(q ; \boldsymbol{i} ; k_{\omega, \ell+1}(r)\right)}\right)^{N_{\omega, i}\left(k_{\omega, \ell}(r)\right) \log v_{\ell} / p_{i}}\right) \\
& \times\left(\prod_{i=1}^{N} \prod_{\ell=1}^{d-1} h_{\omega, i, \ell}\left(q ; \boldsymbol{i} ; k_{\omega, \ell+1}(r)\right)^{\left(1-\frac{1}{\theta_{\ell}} \frac{N_{\omega, i}\left(k_{\omega, \ell+1}(r)\right)}{N_{\omega, i}\left(k_{\omega, \ell}(r)\right)}\right)} N_{\omega, i}\left(k_{\omega, \ell}(r)\right) \log v_{\ell} / p_{i}\right) .
\end{aligned}
$$

The desired result now follows from (5.10), (5.11) and (5.12).

Proposition 5.2 ([KP, Lemma 4.1]). Let $m \in \mathbb{N}$ and $f_{1}, \ldots, f_{m}: \mathbb{N} \rightarrow \mathbb{R}$ satisfy

$$
\max _{i} \sup _{n \in \mathbb{N}}\left|f_{i}(n+1)-f_{i}(n)\right|<\infty .
$$


972

Let $\alpha_{1}, \ldots, \alpha_{m}, \ell_{1}, \ldots, \ell_{m} \in(0, \infty)$. Then

$$
\limsup _{t \rightarrow \infty} \frac{1}{t} \sum_{i=1}^{m}\left(\alpha_{i} f_{i}\left(\left[\frac{t}{L_{i}}\right]\right)-f_{i}\left(\left[\alpha_{i} \frac{t}{L_{i}}\right]\right)\right) \geq 0 .
$$

Proof. See [KP, Lemma 4.1].

Proposition 5.3. Fix $q \in \mathbb{R}$. Let $\omega \in \Omega$ and $\boldsymbol{i} \in D_{\omega}^{\mathbb{N}}$. Then

$$
\underset{r>0}{\limsup } \prod_{i=1}^{N} \prod_{\ell=1}^{d-1} \frac{h_{\omega, i, \ell}\left(q ; \boldsymbol{i} ; k_{\omega, \ell}(r)\right)}{h_{\omega, i, \ell}\left(q ; \boldsymbol{i} ; k_{\omega, \ell+1}(r)\right)} \geq 1
$$

for all $r>0$.

Proof. Let $\omega=\left(\omega_{1}, \omega_{2}, \ldots\right) \in \Omega$ and

$$
\boldsymbol{i}=\mathbf{i}_{1} \mathbf{i}_{2} \ldots \in D_{\omega}^{\mathbb{N}} \quad \text { with } \quad \mathbf{i}_{j}=\left(i_{j, 1}, \ldots, i_{j, d}\right) .
$$

Write $\omega=\left(\mathbf{i}_{1}, \mathbf{i}_{2}, \ldots\right)$. For $i=1, \ldots, n$ and $\ell=1, \ldots, d-1$ define $f_{i, \ell}: \mathbb{N} \rightarrow \mathbb{R}$ by

$$
f_{i, \ell}(n)=\frac{n \log v_{\ell}}{\theta_{\ell}} \log h_{\omega, i, \ell}(q ; \boldsymbol{i} ; n)
$$

Since it is easily seen that $\max _{i, \ell} \sup _{n \in \mathbb{N}}\left|f_{i, \ell}(n+1)-f_{i, \ell}(n)\right|<\infty$ and

$$
\begin{array}{r}
\frac{1}{t} \sum_{i=1}^{N} \sum_{\ell=1}^{d-1}\left(\theta_{\ell} f_{i, \ell}\left(\left[\frac{t}{\log v_{\ell}}\right]\right)-f_{i, \ell}\left(\left[\theta_{\ell} \frac{t}{\log v_{\ell}}\right]\right)\right) \\
=\log \left(\prod_{i=1}^{N} \prod_{\ell=1}^{d-1} \frac{h_{\omega, i, \ell}\left(q ; \boldsymbol{i} ; k_{\omega, \ell}\left(e^{-t}\right)\right)^{w_{\ell}(t)}}{h_{\omega, i, \ell}\left(q ; \boldsymbol{i} ; k_{\omega, \ell+1}\left(e^{-t}\right)\right)^{w_{\ell}\left(\theta_{\ell} t\right)}}\right)
\end{array}
$$

with $w_{\ell}(t):=\left[t / \log v_{\ell}\right] /\left(t / \log v_{\ell}\right)$, Proposition 5.2 shows that

$$
\underset{t \rightarrow \infty}{\limsup } \prod_{i=1}^{N} \prod_{\ell=1}^{d-1} \frac{h_{\omega, i, \ell}\left(q ; \boldsymbol{i} ; k_{\omega, \ell}\left(e^{-t}\right)\right)^{w_{\ell}(t)}}{h_{\omega, i, \ell}\left(q ; \boldsymbol{i} ; k_{\omega, \ell+1}\left(e^{-t}\right)\right)^{w_{\ell}\left(\theta_{\ell} t\right)}} \geq 1 .
$$

Let $\varepsilon>0$. Since $\lim _{t \rightarrow \infty} w_{\ell}(t)=1$, we can choose $t_{0}>0$ such that $1-\varepsilon \leq$ $w_{\ell}(t) \leq 1+\varepsilon$ and $1-\varepsilon \leq w_{\ell}\left(\theta_{\ell} t\right) \leq 1+\varepsilon$ for $\ell=1, \ldots, d-1$ and $t>t_{0}$. It 
now follows from (5.4) and (5.13) that

$$
\begin{aligned}
& 1 \leq \limsup _{t \rightarrow \infty} \prod_{i=1}^{N} \prod_{\ell=1}^{d-1} \frac{h_{\omega, i, \ell}\left(q ; \boldsymbol{i} ; k_{\omega, \ell}\left(e^{-t}\right)\right)^{w_{\ell}(t)}}{h_{\omega, i, \ell}\left(q ; \boldsymbol{i} ; k_{\omega, \ell+1}\left(e^{-t}\right)\right)^{w_{\ell}\left(\theta_{\ell} t\right)}} \\
& \left(\prod_{i, \ell} h_{\omega, i, \ell}\left(q ; i ; k_{\omega, \ell}\left(e^{-t}\right)\right)^{w_{\ell}(t)}\right) \\
& =\limsup _{t_{0}<t, t \rightarrow \infty} \frac{h_{\omega, i, \ell}\left(q ; i ; k_{\omega, \ell}\left(e^{-t}\right)\right)<1}{\left(\prod_{i, \ell} h_{\omega, i, \ell}\left(q ; \boldsymbol{i} ; k_{\omega, \ell+1}\left(e^{-t}\right)\right)^{w_{\ell}\left(\theta_{\ell} t\right)}\right)} \\
& h_{\omega, i, \ell}\left(q ; \mathbf{i} ; k_{\omega, \ell+1}\left(e^{-t}\right)\right)<1 \\
& \left(\prod_{i, \ell} h_{\omega, i, \ell}\left(q ; \boldsymbol{i} ; k_{\omega, \ell}\left(e^{-t}\right)\right)^{w_{\ell}(t)}\right) \\
& \times \frac{h_{\omega, i, \ell}\left(q ; i ; k_{\omega, \ell}\left(e^{-t}\right)\right) \geq 1}{\left(\prod_{i, \ell} h_{\omega, i, \ell}\left(q ; i ; k_{\omega, \ell+1}\left(e^{-t}\right)\right)^{w_{\ell}\left(\theta_{\ell} t\right)}\right)} \\
& h_{\omega, i, \ell}\left(q ; \boldsymbol{i} ; k_{\omega, \ell+1}\left(e^{-t}\right)\right) \geq 1 \\
& \left(\prod_{i=1}^{N} \prod_{\ell=1}^{d-1} h_{\omega, i, \ell}\left(q ; \boldsymbol{i} ; k_{\omega, \ell}\left(e^{-t}\right)\right)\right) \frac{\prod_{i, \ell} h_{\omega, i, \ell}\left(q ; \boldsymbol{i} ; k_{\omega, \ell}\left(e^{-t}\right)\right)^{\varepsilon}}{\prod_{i, \ell, \ell}\left(q ; \boldsymbol{i} ; k_{\omega, \ell}\left(e^{-t}\right)\right) \geq 1} \\
& \leq \limsup _{t_{0}<t, t \rightarrow \infty} \frac{h_{\omega, i, \ell}\left(q ; \mathbf{i} ; k_{\omega, \ell}\left(e^{-t}\right)\right)<1}{\prod h_{\omega, i, \ell}\left(q ; \boldsymbol{i} ; k_{\omega, \ell+1}\left(e^{-t}\right)\right)^{\varepsilon}}
\end{aligned}
$$

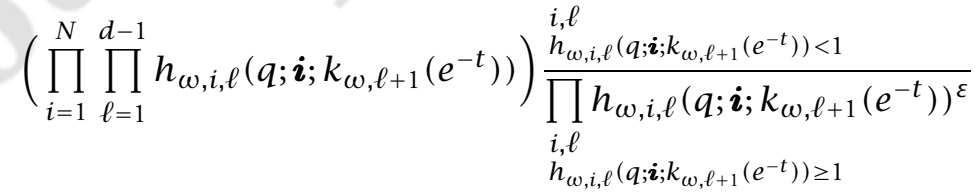

$$
\begin{aligned}
& \leq\left(\frac{h_{\max }(q)}{h_{\min }(q)}\right)^{2 \varepsilon(d-1) N} \limsup _{t \rightarrow \infty} \prod_{i=1}^{N} \prod_{\ell=1}^{d-1} \frac{h_{\omega, i, \ell}\left(q ; \boldsymbol{i} ; k_{\omega, \ell}\left(e^{-t}\right)\right)}{h_{\omega, i, \ell}\left(q ; \boldsymbol{i} ; k_{\omega, \ell+1}\left(e^{-t}\right)\right)}
\end{aligned}
$$

for all $\varepsilon>0$. Letting $\varepsilon \backslash 0$ yields the desired result.

\section{Proposition 5.4.}

(1) For $\mathbb{P}$-almost all $\omega \in \Omega$ we have

$$
\underset{r \backslash 0}{\limsup }\left(\prod_{i=1}^{N} \prod_{\ell=1}^{d-1}\left(\frac{h_{\omega, i, \ell}\left(q ; \boldsymbol{i} ; k_{\omega, \ell}(r)\right)}{h_{\omega, i, \ell}\left(q ; \boldsymbol{i} ; k_{\omega, \ell+1}(r)\right)}\right)^{N_{\omega, i}\left(k_{\omega, \ell}(r)\right) \log v_{\ell} / p_{i}}\right)^{1 /(-\log r)} \geq 1
$$

for all $q \in \mathbb{R}$ and all $\boldsymbol{i} \in D_{\omega}^{\mathbb{N}}$. 
(2) For $\mathbb{P}$-almost all $\omega \in \Omega$ we have, for all $q \in \mathbb{R}$ and all $\boldsymbol{i} \in D_{\omega}^{\mathbb{N}}$,

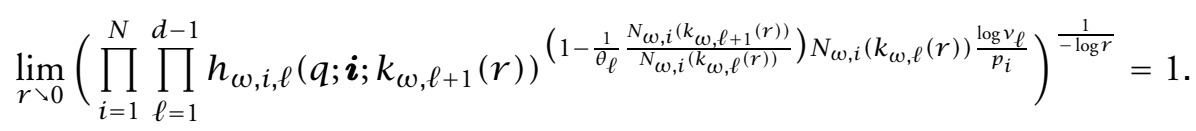

Proof. (1) Since $\lim _{r \backslash 0} \log r / k_{\omega, \ell}(r)=-\log v_{\ell}$ for $\mathbb{P}$-almost all $\omega \in \Omega$ (see Lemma 3.1) and $\lim _{n} N_{\omega, i}(n) / n=p_{i}$ for $\mathbb{P}$-almost all $\omega \in \Omega$, we conclude that

$$
N_{\omega, i}\left(k_{\omega, \ell}(r)\right) \frac{\log v_{\ell}}{p_{i}} \frac{1}{-\log r} \rightarrow 1
$$

for $\mathbb{P}$-almost all $\omega \in \Omega$ and all $\ell$ and all $i$. Next, fix an $\omega \in \Omega$ such that (5.14) holds and let $\boldsymbol{i}=\mathbf{i}_{1} \mathbf{i}_{2} \ldots \in D_{\omega}^{\mathbb{N}}$. Fix $\varepsilon>0$. Since (5.14) holds for all $\ell$ and all $i$, there exists a positive number $r_{0}>0$ such that

$$
1-\varepsilon \leq N_{\omega, i}\left(k_{\omega, \ell}(r)\right) \frac{\log v_{\ell}}{p_{i}} \frac{1}{-\log r} \leq 1+\varepsilon
$$

for all $\ell$ and all $i$ and $r<r_{0}$. Hence, using (5.4) and Proposition 5.3 we conclude that

$$
\begin{aligned}
& \underset{r \backslash 0}{\limsup }\left(\prod_{i=1}^{N} \prod_{\ell=1}^{d-1}\left(\frac{h_{\omega, i, \ell}\left(q ; \boldsymbol{i} ; k_{\omega, \ell}(r)\right)}{h_{\omega, i, \ell}\left(q ; \boldsymbol{i} ; k_{\omega, \ell+1}(r)\right)}\right)^{N_{\omega, i}\left(k_{\omega, \ell}(r)\right) \log v_{\ell} / p_{i}}\right)^{1 /(-\log r)} \\
& \geq \limsup _{r_{0}>r, r>0}\left(\prod_{i, \ell} \quad\left(\frac{h_{\omega, i, \ell}\left(q ; \boldsymbol{i} ; k_{\omega, \ell}(r)\right)}{h_{\omega, i, \ell}\left(q ; \boldsymbol{i} ; k_{\omega, \ell+1}(r)\right)}\right)^{1+\varepsilon}\right) \\
& \frac{h_{\omega, i, \ell}\left(q ; i ; k_{\omega, \ell}(r)\right)}{h_{\omega, i, \ell}\left(q_{i ; i ;}\right)}<1 \\
& \times\left(\prod_{i, \ell}\left(\frac{h_{\omega, i, \ell}\left(q ; \boldsymbol{i} ; k_{\omega, \ell}(r)\right)}{h_{\omega, i, \ell}\left(q ; \boldsymbol{i} ; k_{\omega, \ell+1}(r)\right)}\right)^{1-\varepsilon}\right) \\
& \frac{h_{\omega, i, \ell}\left(q ; ; ; k_{\omega, \ell}(r)\right)}{h_{\omega, i, \ell}\left(q ; i ; k_{\omega, \ell+1}(r)\right)} \geq 1
\end{aligned}
$$

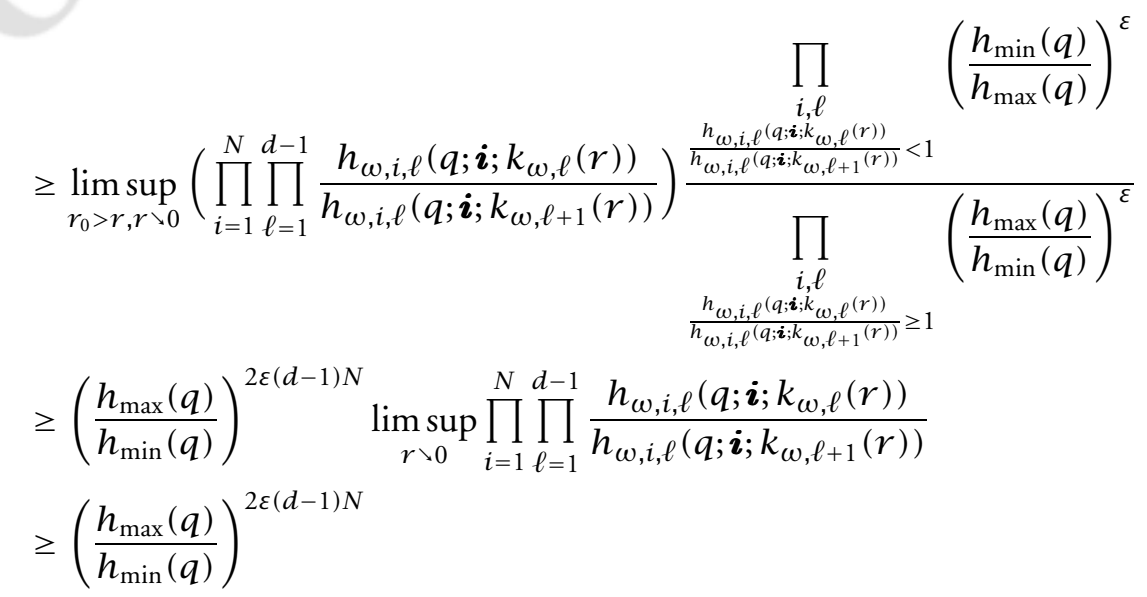


for all $\varepsilon>0$. Letting $\varepsilon>0$ yields the desired result.

(2) Since $\lim _{r \backslash 0} \log r / k_{\omega, \ell}(r)=-\log v_{\ell}$ for $\mathbb{P}$-almost all $\omega \in \Omega$ (see Lemma 3.1) and $\lim _{n} N_{\omega, i}(n) / n=p_{i}$ for $\mathbb{P}$-almost all $\omega \in \Omega$, we conclude that

$$
\left(1-\frac{1}{\theta_{\ell}} \frac{N_{\omega, i}\left(k_{\omega, \ell+1}(r)\right)}{N_{\omega, i}\left(k_{\omega, \ell}(r)\right)}\right) N_{\omega, i}\left(k_{\omega, \ell}(r)\right) \frac{\log v_{\ell}}{p_{i}} \frac{1}{-\log r} \rightarrow 0
$$

for $\mathbb{P}$-almost all $\omega \in \Omega$ and all $\ell$ and all $i$. The desired conclusion now follows immediately from (5.4) and (5.15).

\section{Proposition 5.5.}

(1) For $\mathbb{P}$-almost all $\omega \in \Omega$ we have

$$
\begin{aligned}
& \underset{r \backslash 0}{\limsup }\left(\frac{\mu_{\omega}^{q}\left(Q_{\omega}(\boldsymbol{i}, r)\right)}{\mu_{\omega}\left(Q_{\omega}(\boldsymbol{i}, r)\right)^{q} r^{\beta(q)}}\right)^{1 /(-\log r)}=\limsup _{r \backslash 0} D_{\omega}^{q}(\boldsymbol{i}, r)^{1 /(-\log r)} \geq 1 \\
& \quad \text { for all } q \in \mathbb{R} \text { and all } \boldsymbol{i} \in D_{\omega}^{\mathbb{N}} \text {. } \\
& \text { (2) For } \mathbb{P} \text {-almost all } \omega \in \Omega \text { we have }
\end{aligned}
$$

$$
\underset{r \backslash 0}{\liminf }\left(\frac{\log \mu_{\omega}^{q}\left(Q_{\omega}(\boldsymbol{i}, r)\right)}{\log r}-q \frac{\log \mu_{\omega}\left(Q_{\omega}(\boldsymbol{i}, r)\right)}{\log r}\right) \leq \beta(q)
$$

for all $q \in \mathbb{R}$ and all $\boldsymbol{i} \in D_{\omega}^{\mathbb{N}}$.

Proof. (1) Since $\lim _{r \backslash 0} \log r / k_{\omega, 1}(r)=-\log v_{1}$ for $\mathbb{P}$-almost all $\omega \in \Omega$ (see Lemma 3.1), we immediately conclude that

$$
\lim _{r \succ 0} e^{\left(\beta(q)-\sum_{i=1}^{N} \log v_{1}\left(N_{\omega, i}\left(k_{\omega, 1}(r)\right) /(-\log r)\right) b_{i}(q)\right)}=e^{0}=1
$$

for $\mathbb{P}$-almost all $\omega \in \Omega$, and the desired result follows from this by combining Proposition 5.1 and Proposition 5.4.

(2) This follows immediately from Part (1) by taking logarithms.

Finally, we turn towards the proof of (1.34). In order to prove (1.34) using Proposition 5.5, we need the following version of the mass distribution principle (where balls have been replaced by approximate cubes). This result uses the notation introduced in Proposition 4.7. However, for the benefit of the reader, we repeat the relevant definitions in the statement of Proposition 5.6.

Proposition 5.6. For each $\omega=\left(\omega_{1}, \omega_{2}, \ldots\right), \ell \in\{1, \ldots, d\}$ and $x \in \mathbb{R}$, let

$$
x=\sum_{i=1}^{\infty} \frac{\varepsilon_{\omega, i, \ell}(x)}{n_{\omega_{1}, \ell} n_{\omega_{2}, \ell} \cdots n_{\omega_{i}, \ell}}
$$


with $\varepsilon_{\omega, i, \ell}(x) \in\left\{0,1, \ldots, n_{\omega_{i}, \ell}-1\right\}$, denote the unique non-terminating

$$
\left(\frac{1}{n_{\omega_{1}, \ell}}, \frac{1}{n_{\omega_{1}, \ell} n_{\omega_{2}, \ell}}, \frac{1}{n_{\omega_{1}, \ell} n_{\omega_{2}, \ell} n_{\omega_{3}, \ell}}, \ldots\right) \text {-expansion of } x
$$

For $x=\left(x_{1}, \ldots, x_{d}\right) \in \mathbb{R}^{d}$ write

$$
\boldsymbol{\varepsilon}_{\omega, i}(x)=\left(\varepsilon_{\omega, i, 1}\left(x_{1}\right), \ldots, \varepsilon_{, \omega, i, d}\left(x_{d}\right)\right) \in \Sigma_{\omega_{i}}
$$

(recall that $\Sigma_{\omega_{i}}$ is defined in Section 1.3.1) and put

$$
\boldsymbol{\varepsilon}_{\omega}(x)=\left(\boldsymbol{\varepsilon}_{\omega, 1}(x), \boldsymbol{\varepsilon}_{\omega, 2}(x), \ldots\right) \in \Sigma_{\omega}^{\mathbb{N}}
$$

(recall that $\Sigma_{\omega}^{\mathbb{N}}$ is defined in Section 1.3.1). Let $v$ be a probability measure on $\mathbb{R}^{d}$. Fix $E \subseteq \mathbb{R}^{d}$ and $t>0$. If

$$
\liminf _{r \backslash 0} \frac{\log v\left(Q_{\omega}\left(\varepsilon_{\omega}(x), r\right)\right)}{\log r} \leq t
$$

for all $x \in E$, then

$$
\operatorname{dim}_{H}(E) \leq t
$$

Proof. This result is proved in, for example, [F2] using balls in place of approximate cubes. However, as argued in the proof of Proposition 4.7, the result follows from the proof in [F2] since we, in the definition of the Hausdorff measure, can restrict our attention to covers by approximate cubes.

We can now prove (1.34). We start by introducing some notation and proving a useful auxiliary result-namely, Theorem 5.7. For $\omega \in \Omega$ and $\alpha \in \mathbb{R}$, write

$$
\Delta_{\omega}(\alpha)=\left\{\boldsymbol{i} \in D_{\omega}^{\mathbb{N}} \mid \lim _{r \succ 0} \frac{\log \mu_{\omega}\left(Q_{\omega}(\boldsymbol{i}, r)\right)}{\log r}=\alpha\right\} .
$$

Theorem 5.7. For $\mathbb{P}$-almost all $\omega \in \Omega$ we have that the implication

$$
\Delta_{\omega}(\alpha) \neq \emptyset \Rightarrow f_{\mathrm{H}, \mu_{\omega}}^{\mathrm{sym}}(\alpha) \leq \beta^{*}(\alpha)
$$

holds for all $\alpha \in \mathbb{R}$.

Proof. We first prove that for $\mathbb{P}$-almost all $\omega \in \Omega$ we have

$$
\liminf _{r \backslash 0} \frac{\log \mu_{\omega}^{q}\left(Q_{\omega}(\boldsymbol{i}, r)\right)}{\log r} \leq q \alpha+\beta(q)
$$


for all $q, \alpha \in \mathbb{R}$ and all $\boldsymbol{i} \in \Delta_{\omega}(\alpha)$. Indeed, it follows from Proposition 5.5 that for $\mathbb{P}$-almost all $\omega \in \Omega$ we have

$$
\begin{aligned}
\underset{r \backslash 0}{\liminf } & \frac{\log \mu_{\omega}^{q}\left(Q_{\omega}(\boldsymbol{i}, r)\right)}{\log r}-q \alpha \\
\quad= & \liminf _{r \backslash 0} \frac{\log \mu_{\omega}^{q}\left(Q_{\omega}(\boldsymbol{i}, r)\right)}{\log r}-q \lim _{r \searrow 0} \frac{\log \mu_{\omega}\left(Q_{\omega}(\boldsymbol{i}, r)\right)}{\log r} \\
\quad= & \liminf _{r \backslash 0}\left(\frac{\log \mu_{\omega}^{q}\left(Q_{\omega}(\boldsymbol{i}, r)\right)}{\log r}-q \frac{\log \mu_{\omega}\left(Q_{\omega}(\boldsymbol{i}, r)\right)}{\log r}\right) \leq \beta(q)
\end{aligned}
$$

for all $q, \alpha \in \mathbb{R}$ and all $\boldsymbol{i} \in \Delta_{\omega}(\alpha)$. This proves (5.16).

We conclude from (5.16) and the appropriate version of the mass distribution principle (i.e., Proposition 5.6) that

$$
f_{\mathrm{H}, \mu_{\omega}}^{\mathrm{sym}}(\alpha)=\operatorname{dim}_{\mathrm{H}}\left(\pi_{\omega} \Delta_{\omega}(\alpha)\right) \leq q \alpha+\beta(q) \quad \text { for all } q, \alpha \in \mathbb{R},
$$

whence

$$
f_{\mathrm{H}, \mu_{\omega}}^{\mathrm{sym}}(\alpha) \leq \inf _{q}(q \alpha+\beta(q))=\beta^{*}(\alpha) \quad \text { for all } \alpha .
$$

Next, we prove (1.34). Recall that the numbers $\alpha_{\min }$ and $\alpha_{\max }$ are defined in (1.29).

\section{Theorem 5.8.}

(i) For $\mathbb{P}$-almost all $\omega \in \Omega$ we have

$$
f_{\mathrm{H}, \mu_{\omega}}^{\mathrm{sym}}(\alpha) \leq \beta^{*}(\alpha)
$$

for all $\alpha \in\left(\alpha_{\min }, \alpha_{\max }\right)$.

(ii) For $\mathbb{P}$-almost all $\omega \in \Omega$ we have

$$
\Delta_{\omega}(\alpha)=\emptyset
$$

for all $\alpha \notin\left[\alpha_{\min }, \alpha_{\max }\right]$.

Proof. (i) Let $\omega \in \Omega$ be such that the conclusions in Theorem 4.8 and Theorem 5.7 hold. Fix $\alpha \in\left(\alpha_{\min }, \alpha_{\max }\right)$. It follows from Lemma 4.2 and Theorem 4.8 that $0<\beta^{*}(\alpha) \leq f_{\mathrm{H}, \mu_{\omega}}^{\mathrm{sym}}(\alpha)=\operatorname{dim}_{\mathrm{H}}\left(\pi_{\omega} \Delta_{\omega}(\alpha)\right)$, whence $\Delta_{\omega}(\alpha) \neq \emptyset$, and Theorem 5.7 therefore implies that $f_{\mathrm{H}, \mu_{\omega}}^{\mathrm{sym}}(\alpha) \leq \beta^{*}(\alpha)$.

(ii) Let $\omega \in \Omega$ be such that the conclusion in Theorem 5.7 holds. Fix $\alpha \notin$ $\left[\alpha_{\min }, \alpha_{\max }\right]$, and assume, in order to reach a contradiction, that $\Delta_{\omega}(\alpha) \neq \emptyset$. It therefore follows from Lemma 4.2 and Theorem 5.7 that $0 \leq \operatorname{dim}_{H}\left(\pi_{\omega} \Delta_{\omega}(\alpha)\right)=$ $f_{\mathrm{H}, \mu_{\omega}}^{\mathrm{sym}}(\alpha) \leq \beta^{*}(\alpha)=-\infty$. This gives the desired contradiction. 


\section{Proof of Theorem 1.2}

The purpose of this section is to prove Theorem 1.2.

Proof of Theorem 1.2. Theorem 1.2 follows immediately from Theorem 4.8 and Theorem 5.8 .

\section{Proof of Theorem 1.1}

The purpose of this section is to prove Theorem 1.1. We begin with a small lemma. This lemma says that if $D_{i}$ satisfies the VSSC for all $i=1, \ldots, N$, then the approximate cube $Q_{\omega}(\boldsymbol{i}, r)$ is comparable to the ball $B\left(\pi_{\omega}(\boldsymbol{i}), r\right)$ for all $\omega \in \Omega$ and $\boldsymbol{i} \in D_{\omega}^{\mathbb{N}}$.

Lemma 7.1. Let $\omega \in \Omega, \boldsymbol{i} \in D_{\omega}^{\mathbb{N}}$ and $r>0$. Write $u=\left(\sum_{\ell} \max _{i} n_{i, \ell}^{2}\right)^{1 / 2}$.

(1) If $D_{i}$ satisfies the VSSC for all $i=1, \ldots, N$, then we have $B\left(\pi_{\omega}(i), r\right) \cap$ $K_{\omega} \subseteq Q_{\omega}(i, r)$.

(2) We have $Q_{\omega}(\boldsymbol{i}, r) \subseteq B\left(\pi_{\omega}(\boldsymbol{i}), u r\right)$.

Proof.

(1) Write $\omega=\left(\omega_{1}, \omega_{2}, \ldots\right)$ and $\boldsymbol{i}=\mathbf{i}_{1} \mathbf{i}_{2} \ldots \in D_{\omega}^{\mathbb{N}}$ with $\mathbf{i}_{m}=\left(i_{m, 1}, \ldots, i_{m, d}\right)$ $\in D_{\omega_{m}}$ for all $m$. Let $\pi_{\omega}(\mathbf{j}) \in B(\boldsymbol{\pi}(\boldsymbol{i}), r) \cap K_{\omega}$ with $\mathbf{j}=\mathbf{j}_{1} \mathbf{j}_{2} \ldots \in D_{\omega}^{\mathbb{N}}$ and $\mathbf{j}_{m}=$ $\left(j_{m, 1}, \ldots, j_{m, d}\right) \in D_{\omega_{m}}$ for all $m$. We must now prove that $\pi_{\omega}(\mathbf{j}) \in Q_{\omega}(\boldsymbol{i}, r)$. It is clear that

$$
\pi_{\omega}(\mathbf{j}) \in Q_{\omega}(\mathbf{i}, r)
$$

if and only if

$$
i_{m, 1}=j_{m, 1} \text { for } m=1, \ldots, k_{\omega, 1}(r),
$$

$$
i_{m, d}=j_{m, d} \quad \text { for } m=1, \ldots, k_{\omega, d}(r) .
$$

We will now prove (7.1). To prove (7.1), we assume, in order to obtain a contradiction, that (7.1) is not satisfied. We can therefore choose $\ell_{0} \in\{1, \ldots, d\}$ and $m_{0} \in\left\{1, \ldots, k_{\omega, \ell_{0}}(r)-1\right\}$ such that

$$
i_{m, 1}=j_{m, 1} \quad \text { for } m=1, \ldots, k_{\omega, 1}(r),
$$

$$
\begin{array}{rlrl}
i_{m, \ell_{0}-1} & =j_{m, \ell_{0}-1} & & \text { for } m=1, \ldots, k_{\omega, \ell_{0}-1}(r), \\
i_{m, \ell_{0}} & =j_{m, \ell_{0}} & \text { for } m=1, \ldots, m_{0}, \\
i_{m_{0}+1, \ell_{0}} & \neq j_{m_{0}+1, \ell_{0}} . &
\end{array}
$$

For $\ell=1, \ldots, d$ define projections $T_{\ell}: \mathbb{R}^{d} \rightarrow \mathbb{R}$ by $T_{\ell}\left(x_{1}, \ldots, x_{d}\right)=x_{\ell}$. Since $\pi_{\omega}(\boldsymbol{i}) \in S_{\omega_{1}, \mathbf{i}_{1}} \cdots S_{\omega_{m_{0}+1}, \mathbf{i}_{m_{0}+1}}\left([0,1]^{d}\right), \pi_{\omega}(\mathbf{j}) \in S_{\omega_{1}, \mathbf{j}_{1}} \cdots S_{\omega_{m_{0}+1}, \mathbf{j}_{m_{0}+1}}\left([0,1]^{d}\right)$ 
and $i_{m, \ell_{0}}=j_{m, \ell_{0}}$ for $m=1, \ldots, m_{0}$, we conclude that

$$
\begin{aligned}
& \left|\pi_{\omega}(\boldsymbol{i})-\pi_{\omega}(\mathbf{j})\right| \geq\left|T_{\ell_{0}} \pi_{\omega}(\boldsymbol{i})-T_{\ell_{0}} \pi_{\omega}(\mathbf{j})\right| \\
& \quad \geq \operatorname{dist}\left(T_{\ell_{0}} S_{\omega_{1}, \mathbf{i}_{1}} \cdots S_{\omega_{m_{0}+1}, \mathbf{i}_{m_{0}+1}}\left([0,1]^{d}\right), T_{\ell_{0}} S_{\omega_{1}, \mathbf{j}_{1}} \cdots S_{\omega_{m_{0}+1}, \mathbf{j}_{m_{0}+1}}\left([0,1]^{d}\right)\right) \\
& =\operatorname{dist}\left(T_{\ell_{0}} S_{\omega_{1}, \mathbf{i}_{1}} \cdots S_{\omega_{m_{0}}, \mathbf{i}_{m_{0}}} S_{\omega_{m_{0}+1}, \mathbf{i}_{m_{0}+1}}\left([0,1]^{d}\right),\right. \\
& \left.\quad T_{\ell_{0}} S_{\omega_{1}, \mathbf{i}_{1}} \cdots S_{\omega_{m_{0}}, \mathbf{i}_{m_{0}}} S_{\omega_{m_{0}+1}, \mathbf{j}_{m_{0}+1}}\left([0,1]^{d}\right)\right) \\
& =\frac{1}{n_{\omega_{1}, \ell_{0}} \cdots n_{\omega_{m_{0}}, \ell_{0}}} \operatorname{dist}\left(T_{\ell_{0}} S_{\omega_{m_{0}+1}, \mathbf{i}_{m_{0}+1}}\left([0,1]^{d}\right), T_{\ell_{0}} S_{\omega_{m_{0}+1}, \mathbf{j}_{m_{0}+1}}\left([0,1]^{d}\right)\right) .
\end{aligned}
$$

Since also $D_{\ell_{0}}$ satisfied the VSSC, it follows from (7.2) that $i_{m_{0}+1, \ell}=j_{m_{0}+1, \ell}$ for $\ell=1, \ldots, \ell_{0}-1$ and $\left|i_{m_{0}+1, \ell_{0}}-j_{m_{0}+1, \ell_{0}}\right|>1$, whence

$$
\operatorname{dist}\left(T_{\ell_{0}} S_{\omega_{m_{0}+1}, \mathbf{i}_{m_{0}+1}}\left([0,1]^{d}\right), T_{\ell_{0}} S_{\omega_{m_{0}+1}, \mathbf{j}_{m^{+}+1}}\left([0,1]^{d}\right)\right) \geq \frac{1}{n_{\omega_{m_{0}+1}, \ell_{0}}} .
$$

This inequality, (7.3) and the fact that $m_{0} \leq k_{\omega, \ell_{0}}(r)-1$ imply that

$$
\begin{aligned}
\left|\pi_{\omega}(\boldsymbol{i})-\pi_{\omega}(\mathbf{j})\right| & \geq \frac{1}{n_{\omega_{1}, \ell_{0}} \cdots n_{\omega_{m_{0}}, \ell_{0}} n_{\omega_{m_{0}+1}, \ell_{0}}} \\
& \geq \frac{1}{n_{\omega_{1}, \ell_{0}} \cdots n_{\omega_{m_{0}}, \ell_{0}} n_{\omega_{m_{0}+1}, \ell_{0}} \cdots n_{\omega_{k_{\omega}, \ell_{0}}(r), \ell_{0}}} \geq r .
\end{aligned}
$$

However, since $\pi_{\omega}(\boldsymbol{i}) \in B\left(\pi_{\omega}(\boldsymbol{i}), r\right)$, we clearly have $\left|\pi_{\omega}(\boldsymbol{i})-\pi_{\omega}(\mathbf{j})\right|<r$, which together with (7.4) yields the desired contradiction.

(2) We clearly have

$$
\begin{aligned}
\operatorname{diam} Q_{\omega}(\boldsymbol{i}, r) & =\left(\sum_{\ell}\left(\frac{1}{n_{\omega_{1}, \ell} \cdots n_{\omega_{k_{\omega, \ell}}(r), \ell}}\right)^{2}\right)^{1 / 2} \\
& \leq\left(\sum_{\ell}\left(n_{\omega_{k_{\omega, \ell}(r)+1}, \ell} r\right)^{2}\right)^{1 / 2} \leq u r .
\end{aligned}
$$

As $\pi_{\omega}(\boldsymbol{i}) \in Q_{\omega}(\boldsymbol{i}, r)$, it therefore follows that $Q_{\omega}(\boldsymbol{i}, r) \subseteq B\left(\pi_{\omega}(\boldsymbol{i}), u r\right)$.

Next, we prove Theorem 1.1.

Proof of Theorem 1.1. It follows from Lemma 7.1 that if $\alpha \in \mathbb{R}, \omega \in \Omega$ and $\boldsymbol{i} \in D_{\omega}^{\mathbb{N}}$, then

$$
\lim _{r \backslash 0} \frac{\log \mu_{\omega}\left(Q_{\omega}(\boldsymbol{i}, r)\right)}{\log r}=\alpha \Longleftrightarrow \lim _{r \searrow 0} \frac{\log \mu_{\omega}\left(B\left(\pi_{\omega}(\boldsymbol{i}), r\right)\right)}{\log r}=\alpha .
$$


This and Theorem 1.2 clearly imply the desired result.

\section{WeAKENING OF ASSUMPTION (1.14)}

We will now expand on the remark we made after the statement of Theorem 1.1 concerning our assumption that

$$
v_{1}<v_{2}<\cdots<v_{d}
$$

This assumption is used in the proof of Lemma 3.2. We believe, however, that our results remain true if this assumption is replaced by the following weaker assumption, namely,

$$
v_{1} \leq v_{2} \leq \cdots \leq v_{d}
$$

and we will now outline the necessary adaptation to our arguments. We have the following lemma replacing Lemma 3.2.

Lemma 8.1. Fix $\varepsilon>0$. There is a set $V_{\varepsilon} \subseteq \Omega$ such that

(1) $\mathbb{P}\left(V_{\varepsilon}\right)=1$;

(2) for all $\omega \in V_{\varepsilon}$ there is a real number $r_{\varepsilon, 0}(\omega)>0$ such that

$$
k_{\omega, 1}(r) \geq k_{\omega, 2}\left(r^{1-\varepsilon}\right) \geq \cdots \geq k_{\omega, d}\left(r^{1-(d-1) \varepsilon}\right)
$$

and

$$
k_{\omega, 1}\left(r^{1+(d-1) \varepsilon}\right) \geq k_{\omega, 2}\left(r^{1+(d-2) \varepsilon}\right) \geq \cdots \geq k_{\omega, d}(r)
$$

for all $0<r<r_{\varepsilon, 0}(\omega)$.

Proof. This follows immediately from Lemma 3.1 and the fact that $v_{1} \leq v_{2} \leq$ $\cdots \leq v_{d}$.

Unfortunately, replacing (8.1) by (8.2) we are unable to obtain a precise formula for $\mu_{\omega}(Q(\boldsymbol{i}, r))$ analogous to Lemma 3.3 since we no longer have $k_{\omega, 1}(r) \geq$ $k_{\omega, 2}(r) \geq \cdots \geq k_{\omega, d}(r)$ for sufficiently small $r$. However, using Lemma 8.1 we can prove Lemma 8.2 below (replacing Lemma 3.3), allowing us to estimate $\mu_{\omega}(Q(i, r))$ in the following way.

Lemma 8.2. Let $\varepsilon>0$. Let $\omega=\left(\omega_{1}, \omega_{2}, \ldots\right) \in V_{\varepsilon}$ (where $V_{\varepsilon}$ is defined in Lemma 8.1). For $\boldsymbol{i}=\mathbf{i}_{1} \mathbf{i}_{2} \ldots \in D_{\omega}^{\mathbb{N}}$ with $\mathbf{i}_{j}=\left(i_{j, 1}, \ldots, i_{j, d}\right) \in D_{\omega_{j}}$ and $0<r<r_{\varepsilon, 0}(\omega)$ (where $r_{\varepsilon, 0}(\omega)$ is defined in Lemma 8.1), we have

$$
\begin{aligned}
& \prod_{\ell=1}^{d} \prod_{j=1}^{k_{\omega, \ell}\left(r^{1-(\ell-1) \varepsilon}\right)} p_{\omega_{j}, \ell}\left(i_{j, \ell} \mid i_{j, 1}, \ldots, i_{j, \ell-1}\right) \\
& \quad \leq \mu_{\omega}\left(Q_{\omega}(\boldsymbol{i}, r)\right) \leq \prod_{\ell=1}^{d} \prod_{j=1}^{k_{\omega, \ell}\left(r^{1+(d-\ell) \varepsilon}\right)} p_{\omega_{j}, \ell}\left(i_{j, \ell} \mid i_{j, 1}, \ldots, i_{j, \ell-1}\right) .
\end{aligned}
$$


Proof. This follows immediately from the definition of $\mu_{\omega}$ and the fact that

$$
k_{\omega, 1}(r) \geq k_{\omega, 2}\left(r^{1-\varepsilon}\right) \geq \cdots \geq k_{\omega, d}\left(r^{1-(d-1) \varepsilon}\right)
$$

and

$$
k_{\omega, 1}\left(r^{1+(d-1) \varepsilon}\right) \geq k_{\omega, 2}\left(r^{1+(d-2) \varepsilon}\right) \geq \cdots \geq k_{\omega, d}(r) \quad \text { for all } 0<r<r_{\varepsilon, 0}(\omega),
$$

which follows from Lemma 8.1.

A similar estimate for $\mu_{\omega}^{q}(Q(\boldsymbol{i}, r))$ can also be obtained. We believe that the arguments in this paper can be adapted using the estimates above in place of the precise formula given in Lemmas 3.3 and 3.4. We would often have to work with upper and lower bounds instead of equalities (for example in the proof of Claim 4.6 in Proposition 4.4) and then obtain the desired results by letting $\varepsilon \rightarrow 0$.

We note that the above approach for weakening assumption (1.14) is similar to the arguments presented in [R1].

Acknowledgements. We thank an anonymous referee for suggesting the approach for weakening assumption (1.14) outlined in Section 8. J. M. Fraser acknowledges support from EPSRC.

\section{REFERENCES}

[AN] A.G. ABERCROMBIE AND R. NAIR, On the Hausdorff dimension of certain self-affine sets, Studia Math. 152 (2002), no. 2, 105-124. http://dx.doi.org/10.4064/sm152-2-1. MR1916544 (2003e:28010).

[B] T. Bedford, Crinkly curves, Markov partitions and box dimensions in self-similar sets, Ph.D. dissertation, University of Warwick, 1984.

[BM1] J. BARRAL AND M. MENSI, Gibbs measures on self-affine Sierpinski carpets and their singularity spectrum, Ergodic Theory Dynam. Systems 27 (2007), no. 5, 1419-1443. http://dx.doi.org/10.1017/S0143385706001027. MR2358972 (2009c:37022).

[BM2] _ Multifractal analysis of Birkhoff averages on 'self-affine' symbolic spaces, Nonlinearity 21 (2008), no. 10, 2409-2425. http://dx.doi.org/10.1088/0951-7715/21/10/011. MR2439486 (2009e:37018).

[CM] R. CAWLEY AND R.D. MaUldin, Multifractal decompositions of Moran fractals, Adv. Math. 92 (1992), no. 2, 196-236. http://dx.doi.org/10.1016/0001-8708(92)90064-R. MR 1155465 (93b:58085).

[C] C.D. CUTLER, Measure disintegrations with respect to $\sigma$-stable monotone indices and the pointwise representation of packing dimension, Rend. Circ. Mat. Palermo (2) Suppl. 28 (1992), 319339. Conference at Oberwolfach: Measure Theory, 1990. MR1 183059 (93h:28005).

[DN] Q.-R. DENG AND S.-M. NGAI, Multifractal formalism for self-affine measures with overlaps, Arch. Math. (Basel) 92 (2009), no. 6, 614-625.

http://dx.doi.org/10.1007/s00013-009-2969-9. MR2516167 (2010h:28016).

[F1] K.J. FAlCONER, The Hausdorff dimension of self-affine fractals, Math. Proc. Cambridge Philos. Soc. 103 (1988), no. 2, 339-350. http://dx.doi.org/10.1017/S0305004100064926. MR923687 (89h:28010).

[F2] , Techniques in Fractal Geometry, John Wiley \& Sons Ltd., Chichester, 1997. MR1449135 (99f:28013).

[F3] Generalized dimensions of measures on self-affine sets, Nonlinearity 12 (1999), no. 4, $877-891$.

http://dx.doi.org/10.1088/0951-7715/12/4/308. MR1709826 (2000i:28008). 
[FW] D.-J. FENG AND Y. WANG, A class of self-affine sets and self-affine measures, J. Fourier Anal. Appl. 11 (2005), no. 1, 107-124. http://dx.doi.org/10.1007/s00041-004-4031-4. MR2128947 (2006b:28010).

[GL1] D. GatZOURAS AND S.P. LALley, Statistically self-affine sets: Hausdorff and box dimensions, J. Theoret. Probab. 7 (1994), no. 2, 437-468. http://dx.doi.org/10.1007/BF02214277. MR1270610 (95e:28005).

[GL2] Y. GUI AND W. LI, Hausdorff dimension of subsets with proportional fibre frequencies of the general Sierpinski carpet, Nonlinearity 20 (2007), no. 10, 2353-2364. http://dx.doi.org/10.1088/0951-7715/20/10/005. MR2356114 (2009i:28018).

[GL3] - Hausdorff dimension of fiber-coding sub-Sierpinski carpets, J. Math. Anal. Appl. 331 (2007), no. 1, 62-68. http://dx.doi.org/10.1016/j.jmaa.2006.08.071. MR2305988 (2008g:28042).

[GL4] - A generalized multifractal spectrum of the general Sierpinski carpets, J. Math. Anal. Appl. 348 (2008), no. 1, 180-192. http://dx.doi.org/10.1016/j.jmaa.2008.07.008. MR2449337 (2009f:2801 1).

[GL5] A random version of McMullen-Bedford general Sierpinski carpets and its application, Nonlinearity 21 (2008), no. 8, 1745-1758. http://dx.doi.org/10.1088/0951-7715/21/8/004. MR2425936 (2009i:28019).

[GL6] - Multiscale self-affine Sierpinski carpets, Nonlinearity 23 (2010), no. 3, 495-512. http://dx.doi.org/10.1088/0951-7715/23/3/003. MR2586366 (2011a:28024).

$[\mathrm{H}] \quad \mathrm{H}$. HAASE, A survey on the dimension of measures, Topology, Measures, and Fractals (Warnemünde, Germany, August, 1991), Topology, Measures, and Fractals, Math. Res., vol. 66, Akademie-Verlag, Berlin, 1992, pp. 66-75. MR1226280 (94e:28004).

[JS] T. JORDAN AND K. SIMON, Multifractal analysis of Birkhoff averages for some self-affine IFS, Dyn. Syst. 22 (2007), no. 4, 469-483. http://dx.doi.org/10.1080/14689360701524305. MR2377211 (2009b:37042).

[JR] T. JORDAN AND M. RAMS, Multifractal analysis for Bedford-McMullen carpets, Math. Proc. Cambridge Philos. Soc. 150 (2011), 147-156. http://dx.doi.org/10.1017/S0305004110000472. MR2739077 (2012a:28010).

[KP] R. KENYON AND Y. PERES, Measures of full dimension on affine-invariant sets, Ergodic Theory Dynam. Systems 16 (1996), no. 2, 307-323. http://dx.doi.org/10.1017/S0143385700008828. MR1389626 (98m:28042).

[K] J.F. KING, The singularity spectrum for general Sierpiński carpets, Adv. Math. 116 (1995), no. 1, 1-11. http://dx.doi.org/10.1006/aima.1995.1061. MR1361476 (97d:28008).

[M1] P. MATTILA, Geometry of Sets and Measures in Euclidean Spaces. Fractals and Rectifiability, Cambridge Studies in Advanced Mathematics, vol. 44, Cambridge University Press, Cambridge, 1995. http://dx.doi.org/10.1017/CBO9780511623813. MR1333890 (96h:28006).

[M2] C. MCMullen, The Hausdorff dimension of general Sierpinski carpets, Nagoya Math. J. 96 (1984), 1-9. MR771063 (86h:11061).

[N] O.A. NIELSEN, The Hausdorff and packing dimensions of some sets related to Sierpinski carpets, Canad. J. Math. 51 (1999), no. 5, 1073-1088. http://dx.doi.org/10.4153/CJM-1999-047-4. MR1718652 (2000k:28011).

[NW] T.-J. NI AND Z.-Y. WEN, The $L^{q}$-spectrum of a class of graph directed self-affine measures, Dyn. Syst. 24 (2009), no. 4, 517-536. http://dx.doi.org/10.1080/14689360903143722. MR2573002 (2011c:28012).

[O1] L. Olsen, Self-affine multifractal Sierpinski sponges in $\mathbb{R}^{d}$, Pacific J. Math. 183 (1998), no. 1, 143-199. http://dx.doi.org/10.2140/pjm.1998.183.143. MR1616626 (99c:28028).

[O2] Symbolic and geometric local dimensions of self-affine multifractal Sierpinski sponges in $\mathbb{R}^{d}$, Stoch. Dyn. 7 (2007), no. 1, 37-51. http://dx.doi.org/10.1142/S0219493707001925. MR2303792 (2008c:28012). 
[O3] , Random self-affine multifractal Sierpinski sponges in $\mathbb{R}^{d}$, Monatsh. Math. 162 (2011), no. 1, 89-117. http://dx.doi.org/10.1007/s00605-009-0160-9. MR2747346.

[P] Y. PERES, The self-affine carpets of McMullen and Bedford have infinite Hausdorff measure, Math. Proc. Cambridge Philos. Soc. 116 (1994), no. 3, 513-526. http://dx.doi.org/10.1017/S0305004100072789. MR1291757 (96k:28016).

[PS] Y. PERES AND B. SOlOMYAK, Problems on self-similar sets and self-affine sets: an update, Fractal Geometry and Stochastics, II (Greifswald/Koserow, 1998), Progr. Probab., vol. 46, Birkhäuser, Basel, 2000, pp. 95-106. MR1 785622 (2001 e:28014).

[R1] M. RAMS, On some non-conformal fractals, Nonlinearity 23 (2010), 2423-2428. http://dx.doi.org/10.1088/0951-7715/23/10/004. MR2672681 (2011f:28008).

[R2] R.H. Riedi, An improved multifractal formalism and self-affine measures, Ph.D. dissertation, ETH Zurich, 1993. Diss. ETH No. 10077.

[R3] R.T. Rockafellar, Convex Analysis, Princeton Mathematical Series, vol. 28, Princeton University Press, Princeton, N.J., 1970. MR0274683 (43 \#445).

[T] S. TAKahashi, Dimension spectra of self-affine sets, Israel J. Math. 127 (2002), 1-17. http://dx.doi.org/10.1007/BF02784523. MR1900691 (2003c:28009).

[Y] L.S. YOUNG, Dimension, entropy and Lyapunov exponents, Ergodic Theory Dynamical Systems 2 (1982), no. 1, 109-124. http://dx.doi.org/10.1017/S0143385700009615. MR684248 (84h:58087).

Department of Mathematics

University of St. Andrews

St. Andrews, Fife KY16 9SS, Scotland, U.K.

E-MAIL: jmf32@st-and.ac.uk

E-MAIL: lo@st-and.ac.uk

KEY WORDS AND PHRASES: multifractals, self-affine measures, Sierpinski sponges, Hausdorff dimension, local dimension.

2000 Mathematics SubJeCt Classification: 28 A78.

Received: March 17, 2010; revised: July 12, 2010.

Article electronically published on July 22, 2010. 